\title{
A NUMERICAL STUDY OF SIEGEL THETA SERIES OF VARIOUS DEGREES FOR THE 48-DIMENSIONAL EVEN UNIMODULAR EXTREMAL LATTICES
}

By

\author{
Michio Ozeki
}

\begin{abstract}
Salvati Manni showed that the difference of the Siegel theta series of degree 4 associated with the two even unimodular 48-dimensional extremal lattices is a constant multiple of the cube $J^{3}$ of the Schottky modular form $J$, which is a Siegel cusp form of degree 4 and weight 8 . His result implies that the Siegel theta series of degree up to 3 is unique. But apparently his method does not supply us the process to compute the Fourier coefficients of these series.

In the present paper we show that the Fourier coefficients of the Siegel theta series associated with the even unimodular 48dimensional extremal lattices of degrees 2 and 3 can be computed explicitly, and the Fourier coefficients of the Siegel theta series of degree 4 for those lattices are computed almost explicitly.
\end{abstract}

\section{Introduction}

In [11] we improved the method, which is initiated in [14], to compute the Fourier coefficients of Siegel theta series of various degrees for the class of 32-dimensional even unimodular extremal lattices. Siegel theta series of degree 4 associated with the 32-dimensional even unimodular extremal lattices depend on the individual lattices. In [10] we showed Siegel theta series of degree 4 associated with the five non-isometric 32-dimensional even unimodular extremal lattices constructed from the five doubly even self-dual binary extremal codes are different from each other.

2000 Mathematics Subject Classification: Primary 11F46, Secondary 11E20, 11T71.

Key words and phrases: Theta series with spherical functions, Siegel theta series, 48-dimensional extremal lattices.

Received June 24, 2016.

Revised October 13, 2016. 
In the present paper we first show that the Fourier coefficients of Siegel theta series of degree up to 3 for the even unimodular 48 dimensional extremal lattices can be computed with the help of the so called Hecke-Schöneberg formulas. The method of computation in the present paper together with that of [11] gives a systematic way to compute the Fourier coefficients of Siegel theta series of lower degrees for the class of even unimodular extremal lattices of some tractable dimensions.

Next in degree 4 we show that Siegel theta series associated with the even unimodular 48 dimensional extremal lattice can be expressed as a linear combination of two specified Siegel modular forms. One is the cube $J^{3}$ of the Schottky modular form $J$, which is a Siegel cusp form of weight 8 , and another is (we may call it) a pan theta series $P \Theta_{4,48}(Z)$ in 48 dimension. It turns out that $P \Theta_{4,48}(Z)$ does not depend on the particular extremal lattice. This feature of the present article may be regarded as a more materialization of a result of Salvati Manni [15] Theorem 3, (ii).

In combinatorial number theory or in the geometry of numbers extremal even unimodular lattices are a class of important objects (c.f. [2]). Combinatorial number theorists know the difficulties of constructing such lattices, and they realize that the problem that whether the constructed lattices are isometric or not is much more difficult one. In the dimension 48 case only 4 constructions of even unimodular extremal lattices are known and they are not isometric each other $([8])$.

\section{Basic Definitions}

\subsection{Some Definitions from Lattice Theory}

Let $\mathbf{Z}$ be the ring of rational integers and $\mathbf{R}$ the field of real numbers. A finitely generated $\mathbf{Z}$-module $L$ in $\mathbf{R}^{g}$ with a positive definite metric is called a positive definite quadratic lattice. Since we treat only the positive definite quadratic lattices, we shall omit the adjectives "positive definite quadratic". A lattice $L$ is integral if $L$ satisfies $(\mathbf{x}, \mathbf{y}) \in \mathbf{Z}$ for any $\mathbf{x}, \mathbf{y} \in L$ where $($,$) is$ the bilinear form associated to the metric. Two integral lattices $L_{1}$ and $L_{2}$ are said to be isometric if and only if there exists a bijective linear mapping from $L_{1}$ to $L_{2}$ preserving the metric. The maximal number of linearly independent vectors over $\mathbf{R}$ in $L$ is called the rank of $L$. The dual lattice $L^{\#}$ of $L$ is defined by

$$
L^{\#}=\left\{\mathbf{y} \in L \otimes_{\mathbf{Z}} \mathbf{Q} \mid(\mathbf{x}, \mathbf{y}) \in \mathbf{Z}, \forall \mathbf{x} \in L\right\} .
$$


Here $\mathbf{Q}$ is the field of rational numbers. A lattice $L$ is even if any element $\mathbf{x}$ of $L$ has even norm $(\mathbf{x}, \mathbf{x})$. In an even lattice $L$, we say that $\mathbf{x}$ is a $2 m$-vector if $(\mathbf{x}, \mathbf{x})=2 m$ holds for some natural number $m$. Let $\Lambda_{2 m}(L)$ be the set defined by

$$
\Lambda_{2 m}(L)=\{\mathbf{x} \in L \mid(\mathbf{x}, \mathbf{x})=2 m\}
$$

A lattice $L$ is called unimodular if $L=L^{\#}$. Even unimodular lattices exist only when $n \equiv 0(\bmod 8)$. The minimal norm of a lattice is $\operatorname{Min}(L)=\min _{\mathbf{x} \in L \backslash\{0\}}(\mathbf{x}, \mathbf{x})$. When $L$ is even unimodular of rank $n$ it holds that (conf. [6])

$$
\operatorname{Min}(L) \leq 2\left[\frac{n}{24}\right]+2 .
$$

Such a lattice which attains the above maximum is said to be extremal.

In the present paper we are interested in any even unimodular extremal 48-dimensional lattice. We denote any one of such lattices as $\mathscr{L}_{48}$. We have $\operatorname{Min}\left(\mathscr{L}_{48}\right)=6$. At present we know just four explicite non-isometric even unimodular extremal 48-dimensional lattices [8].

\subsection{Theta Series of One Complex Variable Associated with the Even Unimodular Lattice}

\subsubsection{A Lattice Version}

Let $L$ be an even unimodular lattice of rank $8 k$, then the (ordinary) theta series for $L$ is defined by

$$
\vartheta(z, L)=\sum_{\mathbf{x} \in L} \exp (\pi i(\mathbf{x}, \mathbf{x}) z)
$$

where $z$ is a complex variable with positive imaginary part. This series is rewritten as

$$
\vartheta(z, L)=\sum_{m=0}^{\infty} a(2 m, L) \exp (2 \pi i m z)
$$

where $a(2 m, L)=\left|\Lambda_{2 m}(L)\right|$.

When we consider the lattice $\mathscr{L}_{48}$, then the Fourier expansion of $\mathscr{\vartheta}\left(z, \mathscr{L}_{48}\right)$ is given by 


$$
\begin{aligned}
\theta\left(\tau, \mathscr{L}_{48}\right)= & 1+52416000 q^{6}+39007332000 q^{8}+6609020221440 q^{10} \\
& +437824977408000 q^{12}+\cdots \\
q= & e^{\pi i z}
\end{aligned}
$$

\subsubsection{A Quadratic Form Version}

Let $T$ be a positive definite symmetric square matrix with real entries of size $g$ written by

$$
T=\left(\begin{array}{cccc}
t_{11} & t_{12} / 2 & \cdots & t_{1 g} / 2 \\
t_{12} / 2 & t_{22} & \cdots & t_{2 g} / 2 \\
\vdots & \vdots & \vdots & \vdots \\
t_{1 g} / 2 & t_{2 g} / 2 & \cdots & t_{g g}
\end{array}\right)
$$

then associated with it a quadratic form $Q_{T}[\xi]$ is defined by

$$
Q_{T}[\xi]=Q_{T}\left[\xi_{1}, \ldots, \xi_{g}\right]=\sum_{1 \leq i \leq j \leq g} t_{i j} \xi_{i} \xi_{j}
$$

where $\xi_{1}, \ldots, \xi_{g}$ are real independent variables. The set of all positive definite symmetric matrices with real entries is denoted by $\mathscr{P}_{g}(\mathbf{R})$. Let $G L_{g}(\mathbf{Z})$ be the group of all unimodular square matrices of size $g$. An action of $U \in G L_{g}(\mathbf{Z})$ to an element of $T \in \mathscr{P}_{g}(\mathbf{R})$ is defined by $T \rightarrow U T U^{t}$, where $U^{t}$ is the transposed matrix of $U$. Two elements $T_{1}, T_{2} \in \mathscr{P}_{g}(\mathbf{R})$ are called integrally equivalent if there is a $U \in G L_{g}(\mathbf{Z})$ such that $T_{2}=U T_{1} U^{t}$ holds. This is a well-known equivalence relation.

An element $T \in \mathscr{P}_{g}(\mathbf{R})$ is called semi-integral if the diagonal entries of $2 T$ are all even integers and the off-diagonal entries are integers. The set of such elements is denoted by $\mathscr{P}_{g}^{s}(\mathbf{Z})$. Suppose $T$ is in $\mathscr{P}_{g}^{s}(\mathbf{Z})$, then the theta series for $Q_{T}[\xi]$ is defined by

$$
\vartheta\left(z, Q_{T}\right)=\sum_{\xi_{1}, \ldots, \xi_{g} \in \mathbf{Z}} \exp \left(\pi i z\left(\sum_{1 \leq i \leq j \leq g} t_{i j} \xi_{i} \xi_{j}\right)\right) .
$$

\subsection{Hecke-Schöneberg Formulas Revisited}

In [13] we gave the formulas for the inner product relations among the vectors in a 48-dimensional extremal even unimodular lattice $\mathscr{L}$. Here we reproduce them without proofs. 
Proposition 2.1. Let $\mathscr{L}_{48}$ be an even unimodular 48 dimensional extremal lattice, $\Lambda_{6}=\Lambda_{6}\left(\mathscr{L}_{48}\right)$ and $\boldsymbol{\alpha} \in \mathscr{L}_{48} \otimes \mathbf{R}$, then we have

$$
\begin{gathered}
\sum_{\mathbf{x} \in \Lambda_{6}}(\mathbf{x}, \boldsymbol{a})^{2}=6552000(\boldsymbol{a}, \boldsymbol{\alpha}) \\
\sum_{\mathbf{x} \in \Lambda_{6}}(\mathbf{x}, \boldsymbol{\alpha})^{4}=2358720(\boldsymbol{\alpha}, \boldsymbol{\alpha})^{2} \\
\sum_{\mathbf{x} \in \Lambda_{6}}(\mathbf{x}, \boldsymbol{\alpha})^{6}=1360800(\boldsymbol{\alpha}, \boldsymbol{\alpha})^{3} \\
\sum_{\mathbf{x} \in \Lambda_{6}}(\mathbf{x}, \boldsymbol{\alpha})^{8}=1058400(\boldsymbol{\alpha}, \boldsymbol{\alpha})^{4} \\
\sum_{\mathbf{x} \in \Lambda_{6}}(\mathbf{x}, \boldsymbol{\alpha})^{10}=1020600(\boldsymbol{\alpha}, \boldsymbol{\alpha})^{5} \\
\sum_{\mathbf{x} \in \Lambda_{6}}(\mathbf{x}, \boldsymbol{\alpha})^{14}-\frac{91 \cdot(\boldsymbol{a}, \boldsymbol{\alpha})}{12} \sum_{\mathbf{x} \in \Lambda_{6}}(\mathbf{x}, \boldsymbol{\alpha})^{12}=-7297290 \cdot(\boldsymbol{\alpha}, \boldsymbol{\alpha})^{7}
\end{gathered}
$$

Related to this proposition there is a simple fact, and we state as a lemma:

Lemma 2.2. Let the notations be the same with Proposition 2.1. Then it holds that

$$
\sum_{\mathbf{x} \in \Lambda_{6}}(\mathbf{x}, \boldsymbol{\alpha})^{0}=52416000
$$

Proof. The lefthand side of (2.0) is just the counting of vectors in $\Lambda_{6}$ which equals the righthand side.

Similarly we can derive another formulas.

Proposition 2.3. Let $\mathscr{L}_{48}$ be an even unimodular 48 dimensional extremal lattice and $\Lambda_{8}=\Lambda_{8}\left(\mathscr{L}_{48}\right)$, then we have

$$
\begin{aligned}
\sum_{\mathbf{x} \in \Lambda_{8}}(\mathbf{x}, \boldsymbol{\alpha})^{2} & =6501222000(\boldsymbol{\alpha}, \boldsymbol{\alpha}) \\
\sum_{\mathbf{x} \in \Lambda_{8}}(\mathbf{x}, \boldsymbol{\alpha})^{4} & =3120586560(\boldsymbol{\alpha}, \boldsymbol{\alpha})^{2}
\end{aligned}
$$




$$
\begin{gathered}
\sum_{\mathbf{x} \in \Lambda_{8}}(\mathbf{x}, \boldsymbol{\alpha})^{6}=2400451200(\boldsymbol{\alpha}, \boldsymbol{\alpha})^{3} \\
\sum_{\mathbf{x} \in \Lambda_{8}}(\mathbf{x}, \boldsymbol{\alpha})^{8}=2489356800(\boldsymbol{\alpha}, \boldsymbol{\alpha})^{4} \\
\sum_{\mathbf{x} \in \Lambda_{8}}(\mathbf{x}, \boldsymbol{\alpha})^{10}=3200601600(\boldsymbol{\alpha}, \boldsymbol{\alpha})^{5} \\
\sum_{\mathbf{x} \in \Lambda_{8}}(\mathbf{x}, \boldsymbol{\alpha})^{14}-\frac{91 \cdot(\boldsymbol{\alpha}, \boldsymbol{\alpha})}{9} \sum_{\mathbf{x} \in \Lambda_{8}}(\mathbf{x}, \boldsymbol{\alpha})^{12}=-40683202560 \cdot(\boldsymbol{a}, \boldsymbol{\alpha})^{7}
\end{gathered}
$$

\subsection{Siegel Theta Series}

A Siegel theta series of degree $g(g \geq 2)$ attached to the even unimodular lattice $L$ is defined by

$$
\Theta_{g}(Z, L)=\sum_{\mathbf{x}_{1}, \ldots, \mathbf{x}_{g} \in L} \exp \left(\pi i \sigma\left(\left[\mathbf{x}_{1}, \ldots, \mathbf{x}_{g}\right] Z\right)\right)
$$

where $Z$ is a variable on the Siegel upper-half space of degree $g,\left[\mathbf{x}_{1}, \ldots, \mathbf{x}_{g}\right]$ is a $g$ by $g$ square matrix whose $(i, j)$ entry is $\left(\mathbf{x}_{i}, \mathbf{x}_{j}\right)$ and $\sigma$ is the trace of the matrix.

The Siegel theta series of degree $g$ can be expanded to

$$
\Theta_{g}(Z, L)=\sum_{T \in \hat{\mathscr{P}}_{g}^{s}(\mathbf{Z})} a(T, L) e^{2 \pi i \sigma(T Z)}
$$

Here $\hat{\mathscr{P}}_{g}^{s}(\mathbf{Z})$ is the set of positive semi-definite semi-integral symmetric square matrices of degree $g$, and $a(T, L)=\left|\left\{\left\langle\mathbf{x}_{1}, \ldots, \mathbf{x}_{g}\right\rangle \in L^{g} \mid\left[\mathbf{x}_{1}, \ldots, \mathbf{x}_{g}\right]=2 T\right\}\right|$, and $|X|$ is the cardinality of a set $X$.

We shall say that $2 T$ is represented by the lattice $L$ if $a(T, L) \neq 0$. We quote one importatn property of $a(T, L)$ above as a proposition:

Proposition 2.4. Let $a(T, L)$ be a Fourier coefficient of the Siegel theta series of degree $g$. Then we have

$$
a\left(U T U^{t}, L\right)=a(T, L),
$$

where $U$ is a unimodular matrix of size $g$.

For the proof of this we refer [21], Formula (48).

An easy but important property of Siegel theta series is the following lemma: 
Lemma 2.5. Let $L$ be an even unimodular lattice, and $\Theta_{g}(Z, L)=$ $\sum_{T \in \hat{\mathscr{P}}_{g}^{s}(\mathbf{Z})} a(T, L) e^{2 \pi i \sigma(T Z)}$ be the Siegel theta series of degree $g$ associated with the lattice L. If a $2 T, T \in \hat{\mathscr{P}}_{g}^{s}$ is represented by the lattice $L$, then $2 U T U^{t}$ is also represented by $L$.

Proof. Suppose $2 U T U^{t}$ is not represented by $L$. This means that $a\left(U T U^{t}, L\right)=0$. But by our assumption we know $a(T, L) \neq 0$. This contradicts to Proposition 2.4 .

Let $T$ be a symmetric square matrix of size $g$ written by

$$
T=\left(\begin{array}{cccc}
t_{11} & t_{12} / 2 & \cdots & t_{1 g} / 2 \\
t_{12} / 2 & t_{22} & \cdots & t_{2 g} / 2 \\
\vdots & \vdots & \vdots & \vdots \\
t_{1 g} / 2 & t_{2 g} / 2 & \cdots & t_{g g}
\end{array}\right) .
$$

We will use the convention $T=\left(t_{11}, t_{22}, \ldots, t_{g g}, t_{12}, t_{13}, t_{23}, \ldots, t_{1 g}, t_{2 g}, \ldots, t_{g-1, g}\right)$.

Fact: A Siegel theta series of degree $g$ associated with an even unimodular lattice $L$ of rank $2 k$ ( $2 k$ is a multiple of 8 ) is a modular form of degree $g$ and weight $k$.

\section{Fourier Coefficients of Siegel Theta Series of Degree 2 for $\mathscr{L}_{48}$}

We compute some Fourier coefficients of Siegel theta series of degree 2 for $\mathscr{L}_{48}$. For this we start from the equation

$$
a\left(T, \mathscr{L}_{48}\right)=\sum_{\mathbf{x}_{1}, \mathbf{x}_{2} \in \mathscr{L}_{48},\left[\mathbf{x}_{1}, \mathbf{x}_{2}\right]=2 T} 1, \quad T \in \hat{\mathscr{P}}_{2}^{S}(\mathbf{Z}) .
$$

We prove a neccesary restriction on the inner products among the vectors in $\mathscr{L}_{48}$.

Lemma 3.1. (i) For any two vectors $\mathbf{x}, \mathbf{y} \in \Lambda_{6}\left(\mathscr{L}_{48}\right)$ we have

$$
(\mathbf{x}, \mathbf{y}) \in\{ \pm 6, \pm 3, \pm 2, \pm 1,0\} .
$$

(ii) For any vector $\mathbf{x} \in \Lambda_{6}\left(\mathscr{L}_{48}\right)$ and for any vector $\mathbf{y} \in \Lambda_{8}\left(\mathscr{L}_{48}\right)$ we have

$$
(\mathbf{x}, \mathbf{y}) \in\{ \pm 4, \pm 3, \pm 2, \pm 1,0\} .
$$

(iii) For any two vectors $\mathbf{x}, \mathbf{y} \in \Lambda_{8}\left(\mathscr{L}_{48}\right)$ we have

$$
(\mathbf{x}, \mathbf{y}) \in\{ \pm 8, \pm 5, \pm 4, \pm 3, \pm 2, \pm 1,0\} .
$$


Proof. Proof of (i). By the Schwartzian inequality:

$$
(\mathbf{x}, \mathbf{y})^{2} \leq(\mathbf{x}, \mathbf{x}) \cdot(\mathbf{y}, \mathbf{y})=36
$$

we have

$$
|(\mathbf{x}, \mathbf{y})| \leq 6 .
$$

Suppose $(\mathbf{x}, \mathbf{y})= \pm 5$, then we have $(\mathbf{x} \mp \mathbf{y}, \mathbf{x} \mp \mathbf{y})=2$. This is impossible because $\mathscr{L}_{48}$ does not contain any vector of norm 2 . Suppose $(\mathbf{x}, \mathbf{y})= \pm 4$, then we have $(\mathbf{x} \mp \mathbf{y}, \mathbf{x} \mp \mathbf{y})=4$. This is impossible because $\mathscr{L}_{48}$ does not contain any vector of norm 4.

We omit the proofs of (ii) and (iii).

For $\mathbf{y} \in \Lambda_{6}$ we put $\lambda_{k}(\mathbf{y})=\#\left\{\mathbf{x} \in \Lambda_{6} \mid(\mathbf{x}, \mathbf{y})=k\right\}$. Here $k$ takes the values $\pm 6, \pm 3, \pm 2, \pm 1,0$ by Lemma 3.1,(i). We easily see that $\lambda_{-6}=\lambda_{6}=1, \lambda_{-k}=\lambda_{k}$, $k=1,2,3$. By Equation (2.1) we have

$$
2 \cdot 6^{2} \cdot \lambda_{6}(\mathbf{y})+2 \cdot 3^{2} \cdot \lambda_{3}(\mathbf{y})+2 \cdot 2^{2} \cdot \lambda_{2}(\mathbf{y})+2 \cdot \lambda_{1}(\mathbf{y})=6552000 \cdot 6 .
$$

Likewise from Equations (2.2) and (2.3) we have

$$
2 \cdot 6^{4} \cdot \lambda_{6}(\mathbf{y})+2 \cdot 3^{4} \cdot \lambda_{3}(\mathbf{y})+2 \cdot 2^{4} \cdot \lambda_{2}(\mathbf{y})+2 \cdot \lambda_{1}(\mathbf{y})=2358720 \cdot 6^{2},
$$

and

$$
2 \cdot 6^{6} \cdot \lambda_{6}(\mathbf{y})+2 \cdot 3^{6} \cdot \lambda_{3}(\mathbf{y})+2 \cdot 2^{6} \cdot \lambda_{2}(\mathbf{y})+2 \cdot \lambda_{1}(\mathbf{y})=1360800 \cdot 6^{3} .
$$

Solving these equations we have $\lambda_{3}(\mathbf{y})=36848, \quad \lambda_{2}(\mathbf{y})=1678887, \quad \lambda_{1}(\mathbf{y})=$ 12608784. From the obvious equation:

$$
\lambda_{0}(\mathbf{y})+2 \lambda_{1}(\mathbf{y})+2 \lambda_{2}(\mathbf{y})+2 \lambda_{3}(\mathbf{y})+2 \lambda_{6}(\mathbf{y})=\left|\Lambda_{6}\left(\mathscr{L}_{48}\right)\right|=52416000,
$$

we have $\lambda_{0}(\mathbf{y})=23766960$. We put $T_{0}=(3,3,0), T_{1}=(3,3,1), T_{2}=(3,3,2)$, $T_{3}=(3,3,3)$. Then can conclude that

$$
\begin{aligned}
& a\left(T_{0}, \mathscr{L}_{48}\right)=52416000 \cdot 23766960, \quad a\left(T_{1}, \mathscr{L}_{48}\right)=52416000 \cdot 12608784, \\
& a\left(T_{2}, \mathscr{L}_{48}\right)=52416000 \cdot 1678887, \quad a\left(T_{3}, \mathscr{L}_{48}\right)=52416000 \cdot 36848 .
\end{aligned}
$$

Next for $\mathbf{y} \in \Lambda_{8}$ we put $\mu_{k}(\mathbf{y})=\#\left\{\mathbf{x} \in \Lambda_{6} \mid(\mathbf{x}, \mathbf{y})=k\right\}$. Here $k$ takes the values $\pm 4, \pm 3, \pm 2, \pm 1,0$ by Lemma 3.1,(ii). We see $\mu_{-k}=\mu_{k}, k=1,2,3,4$. Instead of determining $\mu_{k}$ 's we directly compute $a\left(T, \mathscr{L}_{48}\right)$ 's. We begin from Equation $(2.1)$ with $\boldsymbol{a}=\mathbf{y} \in \Lambda_{8}$ :

$$
2 \cdot 4^{2} \mu_{4}(\mathbf{y})+2 \cdot 3^{2} \mu_{3}(\mathbf{y})+2 \cdot 2^{2} \mu_{2}(\mathbf{y})+2 \cdot \mu_{1}(\mathbf{y})=655200 \cdot 8 .
$$


From this we have

$$
\sum_{\mathbf{y} \in \Lambda_{8}}\left(2 \cdot 4^{2} \mu_{4}(\mathbf{y})+2 \cdot 3^{2} \mu_{3}(\mathbf{y})+2 \cdot 2^{2} \mu_{2}(\mathbf{y})+2 \cdot \mu_{1}(\mathbf{y})\right)=655200 \cdot 8 \sum_{\mathbf{y} \in \Lambda_{8}} 1
$$

After expansion the first term of the lefthand side of this equation is

$$
\begin{aligned}
2 \cdot 4^{2} \sum_{\mathbf{y} \in \Lambda_{8}} \mu_{4}(\mathbf{y}) & =2 \cdot 4^{2} \sum_{\mathbf{x} \in \Lambda_{6}, \mathbf{y} \in \Lambda_{8},[\mathbf{x}, \mathbf{y}]=2(3,4,4)} 1 \\
& =2 \cdot 4^{2} a\left((3,4,4), \mathscr{L}_{48}\right) .
\end{aligned}
$$

Similarly we have

$$
\begin{aligned}
2 \cdot 3^{2} \sum_{\mathbf{y} \in \Lambda_{8}} \mu_{3}(\mathbf{y}) & =2 \cdot 3^{2} a\left((3,4,3), \mathscr{L}_{48}\right), \\
2 \cdot 2^{2} \sum_{\mathbf{y} \in \Lambda_{8}} \mu_{2}(\mathbf{y}) & =2 \cdot 2^{2} a\left((3,4,2), \mathscr{L}_{48}\right), \\
2 \cdot \sum_{\mathbf{y} \in \Lambda_{8}} \mu_{1}(\mathbf{y}) & =2 a((3,4,1), L) .
\end{aligned}
$$

After all we have

$$
\begin{aligned}
2 \cdot 4^{2} a & \left((3,4,4), \mathscr{L}_{48}\right)+2 \cdot 3^{2} a\left((3,4,3), \mathscr{L}_{48}\right) \\
& +2 \cdot 2^{2} a\left((3,4,2), \mathscr{L}_{48}\right)+2 a\left((3,4,1), \mathscr{L}_{48}\right) \\
= & 655200 \cdot 8 \cdot 39007332000 .
\end{aligned}
$$

Using Equation (2.2) with $\boldsymbol{\alpha}=\mathbf{y} \in \Lambda_{8}$ we have

$$
\begin{aligned}
2 \cdot 4^{4} a\left((3,4,4), \mathscr{L}_{48}\right)+2 \cdot 3^{4} a\left((3,4,3), \mathscr{L}_{48}\right) \\
\quad+2 \cdot 2^{4} a\left((3,4,2), \mathscr{L}_{48}\right)+2 a\left((3,4,1), \mathscr{L}_{48}\right) \\
=2358720 \cdot 8^{2} \cdot 39007332000,
\end{aligned}
$$

and from $(2.3)$

$$
\begin{aligned}
2 \cdot 4^{6} a & \left((3,4,4), \mathscr{L}_{48}\right)+2 \cdot 3^{6} a\left((3,4,3), \mathscr{L}_{48}\right) \\
& +2 \cdot 2^{6} a\left((3,4,2), \mathscr{L}_{48}\right)+2 a\left((3,4,1), \mathscr{L}_{48}\right) \\
= & 1360800 \cdot 8^{3} \cdot 39007332000
\end{aligned}
$$


and finally from $(2.4)$

$$
\begin{aligned}
2 \cdot 4^{8} a & \left((3,4,4), \mathscr{L}_{48}\right)+2 \cdot 3^{8} a\left((3,4,3), \mathscr{L}_{48}\right) \\
& +2 \cdot 2^{8} a\left((3,4,2), \mathscr{L}_{48}\right)+2 a\left((3,4,1), \mathscr{L}_{48}\right) \\
= & 1058400 \cdot 8^{4} \cdot 39007332000 .
\end{aligned}
$$

These four linear equations are enough to solve $a\left((3,4,4), \mathscr{L}_{48}\right), \ldots, a((3,4,1)$, $\left.\mathscr{L}_{48}\right)$. Indeed we have

$$
a\left((3,4,4), \mathscr{L}_{48}\right)=88000540992000, \quad a\left((3,4,3), \mathscr{L}_{48}\right)=7509379497984000,
$$$$
a\left((3,4,2), \mathscr{L}_{48}\right)=113344696797696000, \quad a\left((3,4,1), \mathscr{L}_{48}\right)=499932945727488000 .
$$

As to $a((3,4,0), L)$ we may utilize a special case of the identity proved in [12]:

$$
\sum_{b} a\left((3,4, b), \mathscr{L}_{48}\right)=a\left(3, \mathscr{L}_{48}\right) a\left(4, \mathscr{L}_{48}\right)=\left|\Lambda_{6}\right| \cdot\left|\Lambda_{8}\right|=52416000 \cdot 39007332000
$$

where $b$ runs over all integers so that the matrix $T=(3,4, b)$ is positive semidefinite. In this case $b= \pm 4, \pm 3, \pm 2, \pm 1,0$. It follows from this identity that

$$
a\left((3,4,0), \mathscr{L}_{48}\right)=802858268983680000 .
$$

Thirdly for $\mathbf{y} \in \Lambda_{8}$ we put $v_{k}(\mathbf{y})=\#\left\{\mathbf{x} \in \Lambda_{8} \mid(\mathbf{x}, \mathbf{y})=k\right\}$. Here $k$ takes the values $\pm 8, \pm 5, \pm 4, \pm 3, \pm 2, \pm 1,0$ by Lemma 3.1,(iii). We see $v_{-k}=v_{k}, k=1,2,3,4,5,8$.

Equation (2.7) leads to

$$
\begin{aligned}
& 2 \cdot 8^{2} v_{8}(\mathbf{y})+2 \cdot 5^{2} v_{5}(\mathbf{y})+2 \cdot 4^{2} v_{4}(\mathbf{y})+2 \cdot 3^{2} v_{3}(\mathbf{y})+2 \cdot 2^{2} v_{2}(\mathbf{y})+2 \cdot v_{1}(\mathbf{y}) \\
& \quad=6501222000 \cdot 8 .
\end{aligned}
$$

By summing the both sides with respect to $\mathbf{y} \in \Lambda_{8}$ we have

$$
\begin{aligned}
\sum_{\mathbf{y} \in \Lambda_{8}}\left[2 \cdot 8^{2} v_{8}(\mathbf{y})+2 \cdot 5^{2} v_{5}(\mathbf{y})+2 \cdot 4^{2} v_{4}(\mathbf{y})+2 \cdot 3^{2} v_{3}(\mathbf{y})\right. \\
\left.\quad+2 \cdot 2^{2} v_{2}(\mathbf{y})+2 \cdot v_{1}(\mathbf{y})\right] \\
=2 \cdot 8^{2} \sum_{\mathbf{y} \in \Lambda_{8}} v_{8}(\mathbf{y})+2 \cdot 5^{2} \sum_{\mathbf{y} \in \Lambda_{8}} v_{5}(\mathbf{y})+2 \cdot 4^{2} \sum_{\mathbf{y} \in \Lambda_{8}} v_{4}(\mathbf{y}) \\
\quad+2 \cdot 3^{2} \sum_{\mathbf{y} \in \Lambda_{8}} v_{3}(\mathbf{y})+2 \cdot 2^{2} \sum_{\mathbf{y} \in \Lambda_{8}} v_{2}(\mathbf{y})+2 \cdot \sum_{\mathbf{y} \in \Lambda_{8}} v_{1}(\mathbf{y}) \\
=6501222000 \cdot 8 \sum_{\mathbf{y} \in \Lambda_{8}} 1 .
\end{aligned}
$$


Thus we have

$$
\begin{aligned}
2 \cdot 8^{2} 39007332000+2 \cdot 5^{2} a\left((4,4,5), \mathscr{L}_{48}\right)+2 \cdot 4^{2} a\left((4,4,4), \mathscr{L}_{48}\right) \\
\quad+2 \cdot 3^{2} a\left((4,4,3), \mathscr{L}_{48}\right)+2 \cdot 2^{2} a\left((4,4,2), \mathscr{L}_{48}\right)+2 a\left((4,4,1), \mathscr{L}_{48}\right) \\
=6501222000 \cdot 8 \cdot 39007332000 .
\end{aligned}
$$

Likewise from Equations $(2.8), \ldots,(2.11)$ we have

$$
\begin{array}{rl}
2 \cdot 8^{4} 3 & 007332000+2 \cdot 5^{4} a\left((4,4,5), \mathscr{L}_{48}\right)+2 \cdot 4^{4} a\left((4,4,4), \mathscr{L}_{48}\right) \\
& +2 \cdot 3^{4} a\left((4,4,3), \mathscr{L}_{48}\right)+2 \cdot 2^{4} a\left((4,4,2), \mathscr{L}_{48}\right)+2 a\left((4,4,1), \mathscr{L}_{48}\right) \\
= & 3120586560 \cdot 8^{2} \cdot 39007332000 \\
2 \cdot 8^{6} 39007332000+2 \cdot 5^{6} a\left((4,4,5), \mathscr{L}_{48}\right)+2 \cdot 4^{6} a\left((4,4,4), \mathscr{L}_{48}\right) & \\
& +2 \cdot 3^{6} a\left((4,4,3), \mathscr{L}_{48}\right)+2 \cdot 2^{6} a\left((4,4,2), \mathscr{L}_{48}\right)+2 a\left((4,4,1), \mathscr{L}_{48}\right) \\
= & 2400451200 \cdot 8^{3} \cdot 39007332000, \\
2 \cdot 8^{8} 39007332000+2 \cdot 5^{8} a\left((4,4,5), \mathscr{L}_{48}\right)+2 \cdot 4^{8} a\left((4,4,4), \mathscr{L}_{48}\right) & +2 \cdot 3^{8} a\left((4,4,3), \mathscr{L}_{48}\right)+2 \cdot 2^{8} a\left((4,4,2), \mathscr{L}_{48}\right)+2 a\left((4,4,1), \mathscr{L}_{48}\right) \\
= & 2489356800 \cdot 8^{4} \cdot 39007332000, \\
2 \cdot 8^{10} 39007332000+2 \cdot 5^{10} a\left((4,4,5), \mathscr{L}_{48}\right)+2 \cdot 4^{10} a\left((4,4,4), \mathscr{L}_{48}\right) \\
\quad+2 \cdot 3^{10} a\left((4,4,3), \mathscr{L}_{48}\right)+2 \cdot 2^{10} a\left((4,4,2), \mathscr{L}_{48}\right)+2 a\left((4,4,1), \mathscr{L}_{48}\right) \\
= & 3200601600 \cdot 8^{5} \cdot 39007332000 .
\end{array}
$$

The above equations are enough to solve $a\left((4,4,5), \mathscr{L}_{48}\right), a\left((4,4,4), \mathscr{L}_{48}\right), \ldots$, $a\left((4,4,1), \mathscr{L}_{48}\right)$ numerically and we have

$$
\begin{aligned}
& a\left((4,4,5), \mathscr{L}_{48}\right)=7509379497984000 \\
& a\left((4,4,4), \mathscr{L}_{48}\right)=799235580046176000 \\
& a\left((4,4,3), \mathscr{L}_{48}\right)=17098857116909568000 \\
& a\left((4,4,2), \mathscr{L}_{48}\right)=121116904578109440000 \\
& a\left((4,4,1), \mathscr{L}_{48}\right)=363048461209534464000 .
\end{aligned}
$$


As to the value of $a\left((4,4,0), \mathscr{L}_{48}\right)$ we use a special case of the identity proved in $[12]$ :

$$
\sum_{b} a\left((4,4, b), \mathscr{L}_{48}\right)=a\left(4, \mathscr{L}_{48}\right) a\left(4, \mathscr{L}_{48}\right)=\left|\Lambda_{8}\right|^{2}=39007332000^{2}
$$

where $b$ runs over all integers so that the matrix $T=(4,4, b)$ is positive semidefinite. In this case $b= \pm 8, \pm 5, \pm 4, \pm 3, \pm 2, \pm 1,0$. Thus we have

$$
a\left((4,4,0), \mathscr{L}_{48}\right)=517430013952014072000 .
$$

As a summary of the computation in this section we give the following table.

Table 1. Fourier coefficients of Siegel-theta series of degree 2 for the lattice $\mathscr{L}_{48}$

\begin{tabular}{|r|c|r|}
\hline$d_{T}$ & $T$ & \multicolumn{1}{|c|}{$a\left(T, \mathscr{L}_{48}\right)$} \\
\hline$* 27$ & $(3,3,3)$ & 1931424768000 \\
32 & $(3,3,2)$ & 88000540992000 \\
35 & $(3,3,1)$ & 660902022144000 \\
$* 36$ & $(3,3,0)$ & 1245768975360000 \\
39 & $(3,4,3)$ & 7509379497984000 \\
44 & $(3,4,2)$ & 113344696797696000 \\
47 & $(3,4,1)$ & 499932945727488000 \\
48 & $(3,4,0)$ & 802858268983680000 \\
$* 48$ & $(4,4,4)$ & 799235580046176000 \\
57 & $(4,4,3)$ & 17098857116909568000 \\
60 & $(4,4,2)$ & 121116904578109440000 \\
63 & $(4,4,1)$ & 363048461209534464000 \\
$* 64$ & $(4,4,0)$ & 517430013952014072000 \\
\hline
\end{tabular}

REMARK 1. In the above table and succeeding Table 3 and Table 5, the star $*$ denotes the imprimitive forms.

\section{Fourier Coefficients of Siegel Theta Series of Degree 3 for $\mathscr{L}_{48}$}

We are going to compute the Fourier coefficients of Siegel theta series of degree 3. To do this we first search good candidates of indeces $T$ of degree 3 by extending an appropriate index $T_{1}$ of degree 2, and next we compute the Fourier coefficients for a group of the extended indeces $T$ by using the Hecke-Schöneberg formulas. 


\subsection{General Notations}

Let $\mathfrak{I}$ be a positive semi-definite semi-integral symmetric matrix of size $s$. Let $a_{1}, \ldots, a_{s}$ be $s$ integers, and we denote by $\left(\mathfrak{I},\left\{a_{1} / 2, \ldots, a_{s} / 2\right\}, 3\right)$ the matrix of size $s+1$ defined by

$$
\left(\begin{array}{cc} 
& a_{1} / 2 \\
\mathfrak{I} & \vdots \\
& a_{s} / 2 \\
a_{1} / 2 \cdots a_{s} / 2 & 3
\end{array}\right)
$$

For an $s$-tuple $\mathbf{x}_{1}, \ldots, \mathbf{x}_{s} \in \Lambda_{6}$ satisfying $\left[\mathbf{x}_{1}, \ldots, \mathbf{x}_{s}\right]=2 \mathfrak{I}$ we will use a subset of $\Lambda_{6}$ defined by

$$
\Lambda_{a_{1}, \ldots, a_{s}}\left(2 \mathfrak{I} ; \mathbf{x}_{1}, \ldots, \mathbf{x}_{s}\right)=\left\{\mathbf{z} \in \Lambda_{6} \mid\left(\mathbf{x}_{i}, \mathbf{z}\right)=a_{i}, i=1, \ldots, s\right\},
$$

and

$$
\lambda_{a_{1}, \ldots, a_{s}}\left(2 \mathfrak{I} ; \mathbf{x}_{1}, \ldots, \mathbf{x}_{s}\right)=\left|\Lambda_{a_{1}, \ldots, a_{s}}\left(2 \mathfrak{I} ; \mathbf{x}_{1}, \ldots, \mathbf{x}_{s}\right)\right|
$$

The Fourier coefficient at $\left(\mathfrak{I},\left\{a_{1} / 2, \ldots, a_{s} / 2\right\}, 3\right)$ is described by

$$
a\left(\left(\mathfrak{I},\left\{a_{1} / 2, \ldots, a_{s} / 2\right\}, 3\right), \mathscr{L}\right)=\sum_{\substack{\mathbf{x}_{1}, \ldots, \mathbf{x}_{s} \in \Lambda_{6} \\\left[\mathbf{x}_{1}, \ldots, \mathbf{x}_{s}\right]=2 \mathfrak{I}}} \lambda_{a_{1}, \ldots, a_{s}}\left(2 \mathfrak{T} ; \mathbf{x}_{1}, \ldots, \mathbf{x}_{s}\right)
$$

\subsection{Extension of Matrices of Degree 2 to Matrices of Degree 3}

The following result is very usefull for judging whether the two ternary positive definite quadratic forms are equivalent or not.

Proposition 4.1 (Schiemann [18]). Two ternary positive definite forms with real coefficients are integrally equivalent if and only if their theta series in one variable are equal.

The next lemma serves to eliminate irrelevant cases for our present search.

Lemma 4.2. Let $\mathscr{L}_{48}$ be any one of even unimodular 48 dimensional extremal lattices, and $T$ be a positive definite semi-integral symmetric matrix of degree $g \geq 3$. If $U T U^{t}$ is reduced in the sense of Minkowski reduction, and some of the diagonal entries of it are 1 or 2 . Then the Fourier coefficient $a\left(T, \mathscr{L}_{48}\right)$ for Siegel theta series of degree $g$ associated with the lattice $\mathscr{L}_{48}$ is zero. 
Proof. If a diagonal entry of the reduced matrix $U T U^{t}$ is 1 or two and $2 U T U^{t}$ is represented by the lattice $\mathscr{L}_{48}$. Then there is a vector $\mathbf{x} \in \mathscr{L}_{48}$ which satisfies $(\mathbf{x}, \mathbf{x})=2$ or 4 . This contradicts to the extremality of the lattice $\mathscr{L}_{48}$. Thus we have $a\left(U T U^{t}, \mathscr{L}_{48}\right)=0$ and by Proposition $2.4 a\left(T, \mathscr{L}_{48}\right)=0$.

Notation. Since we will use the Minkowski reduced form of the $T$, where $T$ is a positive definite semi-integral symmetric matrix of degree $g \geq 3$, we use $\operatorname{MR}(T)$ to denote the Minkowski reduced form of $T$. For the Minkowski reduction one may refer [7], [22], [23]. For the degrees $g=3,4$ of $T$ the tables of Minkowski reduced form of $T$ are available at [1], [9].

\subsubsection{First Case}

Let

$$
T_{2,1}=\left(\begin{array}{cc}
3 & 3 / 2 \\
3 / 2 & 3
\end{array}\right)
$$

be the positive definite symmetric semi-integral matrix of size 2 .

First we explore all possible pairs of integers $a, b$ under the conditions:

(i) $\left(T_{2,1},\{a / 2, b / 2\}, 3\right)$ is positive semi-definite,

(ii) under the unimodular transformation

$$
\left(T_{2,1},\{a / 2, b / 2\}, 3\right) \mapsto U^{t}\left(T_{2,1},\{a / 2, b / 2\}, 3\right) U,
$$

the minimal value of the non-zero diagonal entries of the resulting matrix is reduced to 3 .

REMARK 2. In view of Lemma 4.2 it is legitimate to impose the condition (ii) in computing the Fourier coefficients $a\left(\left(T_{2,1},\{a / 2, b / 2\}, 3\right), \mathscr{L}_{48}\right)$.

The pair of integers $\langle a, b\rangle$ satisfying the conditions (i), (ii) are grouped into the sets according to the determinant of $2\left(T_{2,1},\{a / 2, b / 2\}, 3\right)$ and the equivalence by the unimodular transformations. In the ternary quadratic forms $\operatorname{det}\left(2\left(T_{2,1},\{a / 2, b / 2\}, 3\right)\right) / 2$ is called the discriminant of the matrix $T=$ $\left(T_{2,1},\{a / 2, b / 2\}, 3\right)$ (c.f. Section 3.1 in [11]). We denote it by $d$. We write $C_{d}\left(T_{2,1},\langle a, b\rangle\right)$ to denote the set of ordered pairs $\left\langle a^{\prime}, b^{\prime}\right\rangle$ such that $\operatorname{det}\left(2\left(T_{2,1}\right.\right.$, $\{a / 2, b / 2\}, 3)) / 2=\operatorname{det}\left(2\left(T_{2,1},\left\{a^{\prime} / 2, b^{\prime} / 2\right\}, 3\right)\right) / 2=d$ and $\left(T_{2,1},\{a / 2, b / 2\}, 3\right)$ is equivalent to $\left(T_{2,1},\left\{a^{\prime} / 2, b^{\prime} / 2\right\}, 3\right)$. Most cases of $C_{d}\left(T_{2,1},\langle a, b\rangle\right)$ are divided into the two subsets $C_{d}^{+}\left(T_{2,1},\langle a, b\rangle\right)$ and the subset obtained from $C_{d}^{+}\left(T_{2,1},\langle a, b\rangle\right)$ by multiplying each member of each pair by -1 . The convention to use $C_{d}^{+}(\cdots)$ will 
be used at later occasions. The total sets thus defined are called admissible sets with respect to $T_{2,1} . C_{81}\left(T_{2,1},\langle 0,0\rangle\right)=\{\langle 0,0\rangle\}$ and

$$
\begin{aligned}
& C_{78}^{+}\left(T_{2,1},\langle 1,1\rangle\right)=\{\langle 1,1\rangle,\langle 1,0\rangle,\langle 0,1\rangle\}, \\
& C_{72}^{+}\left(T_{2,1},\langle 2,1\rangle\right)=\{\langle 2,1\rangle,\langle 1,2\rangle,\langle 1,-1\rangle\}, \\
& C_{69}^{+}\left(T_{2,1},\langle 2,2\rangle\right)=\{\langle 2,2\rangle,\langle 2,0\rangle,\langle 0,2\rangle\}, \\
& C_{60}^{+}\left(T_{2,1},\langle 3,2\rangle\right)=\{\langle 3,2\rangle,\langle 3,1\rangle,\langle 2,3\rangle,\langle 2,-1\rangle,\langle 1,3\rangle,\langle 1,-2\rangle\}, \\
& C_{54}^{+}\left(T_{2,1},\langle 3,3\rangle\right)=\{\langle 3,3\rangle,\langle 3,0\rangle,\langle 0,3\rangle\}, \\
& C_{0}^{+}\left(T_{2,1},\langle 6,3\rangle\right)=\{\langle 6,3\rangle,\langle 3,6\rangle,\langle 3,-3\rangle\} .
\end{aligned}
$$

REMARK 3. We should keep in mind that each set in the above determines a unique reduced ternary quadratic form. For instance $\left(T_{2,1},\{0,0\}, 3\right)$ is integrally equivalent to the reduced ternary quadratic form $(3,3,3,3,0,0)$ in Table 2 of Section 4.2 .5 below. This rule applies even for degree 4 extensions. See Table 2 and Table 4 below.

Proposition 4.3. For a fixed pair $\mathbf{x}_{1}, \mathbf{x}_{2} \in \Lambda_{6}$ which satisfy $\left[\mathbf{x}_{1}, \mathbf{x}_{2}\right]=2 T_{2,1}$ it can be verified that for any $\mathbf{y} \in \Lambda_{6}$ the pairs of values $\langle a, b\rangle$ such that $\left(\mathbf{x}_{1}, \mathbf{y}\right)=a$ and $\left(\mathbf{x}_{2}, \mathbf{y}\right)=b$ fall in one of $C_{81}\left(T_{2,1},\langle 0,0\rangle\right), C_{78}\left(T_{2,1},\langle 1,1\rangle\right), \ldots$, $C_{0}\left(T_{2,1},\langle 6,3\rangle\right)$.

Proof. This statement is verified by a numerical search with the help of Lemmas 3.1 and 4.2. We omit the details.

For a later purpose we use the cardinalities of the sets of pairs above as $c_{81}\left(T_{2,1}\right)=\left|C_{81}\left(T_{2,1},\langle 0,0\rangle\right)\right|=1, \ldots, c_{0}\left(T_{2,1}\right)=\left|C_{0}\left(T_{2,1},\langle 6,3\rangle\right)\right|=6$.

\subsubsection{Second Case}

Let

$$
T_{2,2}=\left(\begin{array}{ll}
3 & 1 \\
1 & 3
\end{array}\right)
$$

be another positive definite symmetric semi-integral matrix of size 2. This time we seek the pairs of integers $\langle a, b\rangle$ which satisfy the conditions (i) and (ii) but $T_{2,1}$ is replaced by $T_{2,2}$. 
The following are all the possible sets. $C_{96}\left(T_{2,2},\langle 0,0\rangle\right)=\{\langle 0,0\rangle\}$ and

$$
\begin{aligned}
C_{93}^{+}\left(T_{2,2},\langle 0,1\rangle\right) & =\{\langle 0,1\rangle,\langle 1,0\rangle\}, \\
C_{92}^{+}\left(T_{2,2},\langle 1,1\rangle\right) & =\{\langle 1,1\rangle\}, \\
C_{88}^{+}\left(T_{2,2},\langle 1,-1\rangle\right) & =\{\langle 1,-1\rangle\}, \\
C_{85}^{+}\left(T_{2,2},\langle 2,1\rangle\right) & =\{\langle 2,1\rangle,\langle 1,2\rangle\}, \\
C_{84}^{+}\left(T_{2,2},\langle 2,0\rangle\right) & =\{\langle 2,0\rangle,\langle 0,2\rangle\}, \\
C_{80}^{+}\left(T_{2,2},\langle 2,2\rangle\right) & =\{\langle 2,2\rangle\}, \\
C_{77}^{+}\left(T_{2,2},\langle 2,-1\rangle\right) & =\{\langle 2,-1\rangle,\langle 1,-2\rangle\}, \\
C_{72}^{+}\left(T_{2,2},\langle 3,1\rangle\right) & =\{\langle 3,1\rangle,\langle 1,3\rangle\}, \\
C_{69}^{+}\left(T_{2,2},\langle 3,2\rangle\right) & =\{\langle 3,2\rangle,\langle 3,0\rangle,\langle 2,3\rangle,\langle 0,3\rangle\}, \\
C_{64}^{+}\left(T_{2,2},\langle 2,-2\rangle\right) & =\{\langle 2,-2\rangle\}, \\
C_{60}^{+}\left(T_{2,2},\langle 3,3\rangle\right) & =\{\langle 3,3\rangle,\langle 3,-1\rangle,\langle 1,-3\rangle\}, \\
C_{0}^{+}\left(T_{2,2},\langle 6,2\rangle\right) & =\{\langle 6,2\rangle,\langle 2,6\rangle\},
\end{aligned}
$$

Proposition 4.4. For a fixed pair $\mathbf{x}_{1}, \mathbf{x}_{2} \in \Lambda_{6}$ which satisfy $\left[\mathbf{x}_{1}, \mathbf{x}_{2}\right]=2 T_{2,2}$ it can be verified that for any $\mathbf{y} \in \Lambda_{6}$ the pairs of values $\langle a, b\rangle$ such that $\left(\mathbf{x}_{1}, \mathbf{y}\right)=a$ and $\left(\mathbf{x}_{2}, \mathbf{y}\right)=b$ fall in one of $C_{96}\left(T_{2,2},\langle 0,0\rangle\right), C_{92}\left(T_{2,2},\langle 1,1\rangle\right), \ldots$, $C_{0}\left(T_{2,2},\langle 6,2\rangle\right)$.

\subsubsection{Third Case}

We put

$$
T_{2,3}=\left(\begin{array}{cc}
3 & 1 / 2 \\
1 / 2 & 3
\end{array}\right)
$$

which is a positive definite symmetric semi-integral matrix of size 2 .

We search the pairs of integers $\langle a, b\rangle$ which satisfy the conditions (i) and (ii) but $T_{2,1}$ is replaced by $T_{2,3}$.

We present only the results.

$$
\begin{gathered}
C_{105}\left(T_{2,3},\langle 0,0\rangle\right)=\{\langle 0,0\rangle\} \text { and } \\
C_{102}^{+}\left(T_{2,3},\langle 1,0\rangle\right)=\{\langle 1,0\rangle,\langle 0,1\rangle\}, \\
C_{100}^{+}\left(T_{2,3},\langle 1,1\rangle\right)=\{\langle 1,1\rangle\},
\end{gathered}
$$




$$
\begin{aligned}
C_{98}^{+}\left(T_{2,3},\langle 1,-1\rangle\right) & =\{\langle 1,-1\rangle\}, \\
C_{93}^{+}\left(T_{2,3},\langle 2,0\rangle\right) & =\{\langle 2,0\rangle,\langle 0,2\rangle\}, \\
C_{92}^{+}\left(T_{2,3},\langle 2,1\rangle\right) & =\{\langle 2,1\rangle,\langle 1,2\rangle\}, \\
C_{88}^{+}\left(T_{2,3},\langle 2,-1\rangle\right) & =\{\langle 2,-1\rangle,\langle 1,-2\rangle\}, \\
C_{85}^{+}\left(T_{2,3},\langle 2,2\rangle\right) & =\{\langle 2,2\rangle\}, \\
C_{78}^{+}\left(T_{2,3},\langle 3,1\rangle\right) & =\{\langle 3,1\rangle,\langle 3,0\rangle,\langle 1,3\rangle,\langle 0,3\rangle\}, \\
C_{77}^{+}\left(T_{2,3},\langle 2,-2\rangle\right) & =\{\langle 2,-2\rangle\}, \\
C_{72}^{+}\left(T_{2,3},\langle 3,2\rangle\right) & =\{\langle 3,2\rangle,\langle 3,-1\rangle,\langle 2,3\rangle,\langle 1,-3\rangle\}, \\
C_{60}^{+}\left(T_{2,3},\langle 3,3\rangle\right) & =\{\langle 3,3\rangle,\langle 3,-2\rangle,\langle 2,-3\rangle\}, \\
C_{0}^{+}\left(T_{2,3},\langle 6,1\rangle\right) & =\{\langle 6,1\rangle,\langle 1,6\rangle\} .
\end{aligned}
$$

Proposition 4.5. For a fixed pair $\mathbf{x}_{1}, \mathbf{x}_{2} \in \Lambda_{6}$ which satisfy $\left[\mathbf{x}_{1}, \mathbf{x}_{2}\right]=2 T_{2,3}$ it can be verified that for any $\mathbf{y} \in \Lambda_{6}$ the pairs of values $\langle a, b\rangle$ such that $\left(\mathbf{x}_{1}, \mathbf{y}\right)=a$ and $\left(\mathbf{x}_{2}, \mathbf{y}\right)=b$ fall in one of $C_{105}\left(T_{2,3},\langle 0,0\rangle\right), C_{102}\left(T_{2,3},\langle 1,0\rangle\right), \ldots$, $C_{0}\left(T_{2,3},\langle 6,1\rangle\right)$.

\subsubsection{Fourth Case}

We put

$$
T_{2,4}=\left(\begin{array}{ll}
3 & 0 \\
0 & 3
\end{array}\right)
$$

which is a positive definite symmetric half-integral matrix of size 2 .

We search the pairs of integers $\langle a, b\rangle$ which satisfy the conditions (i) and (ii) but $T_{2,1}$ is replaced by $T_{2,4}$.

$$
\begin{aligned}
C_{108}\left(T_{2,4},\langle 0,0\rangle\right)=\{\langle 0,0\rangle\} \text { and } \\
C_{105}^{+}\left(T_{2,4},\langle 1,0\rangle\right)=\{\langle 1,0\rangle,\langle 0,1\rangle\}, \\
C_{102}^{+}\left(T_{2,4},\langle 1,1\rangle\right)=\{\langle 1,1\rangle,\langle 1,-1\rangle\}, \\
C_{96}^{+}\left(T_{2,4},\langle 2,0\rangle\right)=\{\langle 2,0\rangle,\langle 0,2\rangle\}, \\
C_{93}^{+}\left(T_{2,4},\langle 2,1\rangle\right)=\{\langle 2,1\rangle,\langle 2,-1\rangle,\langle 1,2\rangle,\langle 1,-2\rangle\}, \\
C_{84}^{+}\left(T_{2,4},\langle 2,2\rangle\right)=\{\langle 2,2\rangle,\langle 2,-2\rangle\},
\end{aligned}
$$




$$
\begin{aligned}
& C_{81}^{+}\left(T_{2,4},\langle 3,0\rangle\right)=\{\langle 3,0\rangle,\langle 0,3\rangle\}, \\
& C_{78}^{+}\left(T_{2,4},\langle 3,1\rangle\right)=\{\langle 3,1\rangle,\langle 3,-1\rangle,\langle 1,3\rangle,\langle 1,-3\rangle\}, \\
& C_{69}^{+}\left(T_{2,4},\langle 3,2\rangle\right)=\{\langle 3,2\rangle,\langle 3,-2\rangle,\langle 2,3\rangle,\langle 2,-3\rangle\}, \\
& C_{54}^{+}\left(T_{2,4},\langle 3,3\rangle\right)=\{\langle 3,3\rangle,\langle 3,-3\rangle\}, \\
& C_{0}^{+}\left(T_{2,4},\langle 6,0\rangle\right)=\{\langle 6,0\rangle,\langle 0,6\rangle\} .
\end{aligned}
$$

Proposition 4.6. For a fixed pair $\mathbf{x}_{1}, \mathbf{x}_{2} \in \Lambda_{6}$ which satisfy $\left[\mathbf{x}_{1}, \mathbf{x}_{2}\right]=2 T_{2,4}$ it can be verified that for any $\mathbf{y} \in \Lambda_{6}$ the pairs of values $\langle a, b\rangle$ such that $\left(\mathbf{x}_{1}, \mathbf{y}\right)=a$ and $\left(\mathbf{x}_{2}, \mathbf{y}\right)=b$ fall in one of $C_{108}\left(T_{2,4},\langle 0,0\rangle\right), C_{105}\left(T_{2,4},\langle 1,0\rangle\right), \ldots$, $C_{0}\left(T_{2,4},\langle 6,0\rangle\right)$.

\subsubsection{An Assembled Table of Ternary Quadratic Forms}

Table 2. Table of extended ternary quadratic forms and its reduced forms

\begin{tabular}{|l|l|c|}
\hline & \multicolumn{1}{|c|}{ extended ternary forms } & reduced ternary forms \\
\hline$A_{1}$ & $C_{54}\left(T_{2,1}\right), C_{54}\left(T_{2,4}\right)$ & $(3,3,3,3,3,0)$ \\
\hline$A_{2}$ & $C_{60}\left(T_{2,1}\right), C_{60}\left(T_{2,2}\right), C_{60}\left(T_{2,3}\right)$ & $(3,3,3,3,2,-1)$ \\
\hline$A_{3}$ & $C_{64}\left(T_{2,2}\right)$ & $(3,3,3,2,2,-2)$ \\
\hline$A_{4}$ & $C_{69}\left(T_{2,1}\right), C_{69}\left(T_{2,4}\right)$ & $(3,3,3,3,2,0)$ \\
\hline$A_{5}$ & $C_{72}\left(T_{2,1}\right), C_{72}\left(T_{2,3}\right)$ & $(3,3,3,3,1,-1)$ \\
\hline$A_{6}$ & $C_{77}\left(T_{2,2}\right), C_{77}\left(T_{2,3}\right)$ & $(3,3,3,2,2,-1)$ \\
\hline$A_{7}$ & $C_{78}\left(T_{2,1}\right), C_{78}\left(T_{2,3}\right), C_{78}\left(T_{2,4}\right)$ & $(3,3,3,3,1,0)$ \\
\hline$A_{8}$ & $C_{80}\left(T_{2,2}\right)$ & $(3,3,3,2,2,2)$ \\
\hline$A_{9}$ & $C_{81}\left(T_{2,1}\right), C_{81}\left(T_{2,4}\right)$ & $(3,3,3,3,0,0)$ \\
\hline$A_{10}$ & $C_{84}\left(T_{2,2}\right), C_{84}\left(T_{2,4}\right)$ & $(3,3,3,2,2,0)$ \\
\hline$A_{11}$ & $C_{85}\left(T_{2,2}\right), C_{85}\left(T_{2,3}\right)$ & $(3,3,3,2,2,1)$ \\
\hline$A_{12}$ & $C_{88}\left(T_{2,2}\right), C_{88}\left(T_{2,3}\right)$ & $(3,3,3,2,1,-1)$ \\
\hline$A_{13}$ & $C_{92}\left(T_{2,2}\right), C_{92}\left(T_{2,3}\right)$ & $(3,3,3,2,1,1)$ \\
\hline$A_{14}$ & $C_{93}\left(T_{2,2}\right), C_{93}\left(T_{2,3}\right), C_{93}\left(T_{2,4}\right)$ & $(3,3,3,2,1,0)$ \\
\hline$A_{15}$ & $C_{96}\left(T_{2,2}\right), C_{96}\left(T_{2,4}\right)$ & $(3,3,3,2,0,0)$ \\
\hline & & \\
\hline
\end{tabular}


Table 2 (continued)

\begin{tabular}{|l|l|c|}
\hline & extended ternary forms & reduced ternary forms \\
\hline$A_{16}$ & $C_{98}\left(T_{2,3}\right)$ & $(3,3,3,1,1,-1)$ \\
\hline$A_{17}$ & $C_{100}\left(T_{2,3}\right)$ & $(3,3,3,1,1,1)$ \\
\hline$A_{18}$ & $C_{102}\left(T_{2,3}\right), C_{102}\left(T_{2,4}\right)$ & $(3,3,3,1,1,0)$ \\
\hline$A_{19}$ & $C_{105}\left(T_{2,3}\right), C_{105}\left(T_{2,4}\right)$ & $(3,3,3,1,0,0)$ \\
\hline$A_{20}$ & $C_{108}\left(T_{2,4}\right)$ & $(3,3,3,0,0,0)$ \\
\hline
\end{tabular}

\subsection{Computing the Fourier Coefficients of Siegel Theta Series of Degree 3} for $L$

\subsubsection{The First Case (in Detail)}

For a fixed pair $\mathbf{x}_{1}, \mathbf{x}_{2} \in \Lambda_{6}$ which satisfy $\left[\mathbf{x}_{1}, \mathbf{x}_{2}\right]=2 T_{2,1}$, we set

$$
\Lambda_{a, b}\left(2 T_{2,1} ; \mathbf{x}_{1}, \mathbf{x}_{2}\right)=\left\{\mathbf{z} \in \Lambda_{6} \mid\left(\mathbf{x}_{1}, \mathbf{z}\right)=a,\left(\mathbf{x}_{2}, \mathbf{z}\right)=b\right\},
$$

and

$$
\lambda_{a, b}\left(2 T_{2,1} ; \mathbf{x}_{1}, \mathbf{x}_{2}\right)=\left|\Lambda_{a, b}\left(2 T_{2,1} ; \mathbf{x}_{1}, \mathbf{x}_{2}\right)\right|
$$

We take $\boldsymbol{\alpha}=u \mathbf{x}_{1}+v \mathbf{x}_{2}$, where $u$ and $v$ are real independent variables, then we see that for $\mathbf{y} \in \Lambda_{6}$

$$
(\mathbf{y}, \boldsymbol{a})=a u+b v .
$$

Then by the formula (2.1) we have

$$
\begin{aligned}
\sum_{\mathbf{y} \in \Lambda_{6}} \lambda_{a, b}\left(2 T_{2,1} ; \mathbf{x}_{1}, \mathbf{x}_{2}\right)(a u+b v)^{2} & \\
= & \sum_{\langle a, b\rangle \in C_{78}\left(T_{2,1},\langle 1,1\rangle\right)} \lambda_{a, b}\left(2 T_{2,1} ; \mathbf{x}_{1}, \mathbf{x}_{2}\right)(a u+b v)^{2} \\
& +\sum_{\langle a, b\rangle \in C_{72}\left(T_{2,1},\langle 2,1\rangle\right)} \lambda_{a, b}\left(2 T_{2,1} ; \mathbf{x}_{1}, \mathbf{x}_{2}\right)(a u+b v)^{2} \\
& +\sum_{\langle a, b\rangle \in C_{69}\left(T_{2,1},\langle 2,2\rangle\right)} \lambda_{a, b}\left(2 T_{2,1} ; \mathbf{x}_{1}, \mathbf{x}_{2}\right)(a u+b v)^{2} \\
& +\sum_{\langle a, b\rangle \in C_{60}\left(T_{2,1},\langle 3,2\rangle\right)} \lambda_{a, b}\left(2 T_{2,1} ; \mathbf{x}_{1}, \mathbf{x}_{2}\right)(a u+b v)^{2}
\end{aligned}
$$




$$
\begin{aligned}
& +\sum_{\langle a, b\rangle \in C_{54}\left(T_{2,1},\langle 3,3\rangle\right)} \lambda_{a, b}\left(2 T_{2,1} ; \mathbf{x}_{1}, \mathbf{x}_{2}\right)(a u+b v)^{2} \\
& +\sum_{\langle a, b\rangle \in C_{0}\left(T_{2,1},\langle 6,3\rangle\right)} \lambda_{a, b}\left(2 T_{2,1} ; \mathbf{x}_{1}, \mathbf{x}_{2}\right)(a u+b v)^{2} \\
& =6552000(\boldsymbol{a}, \boldsymbol{\alpha}) \\
& =6552000\left(6 u^{2}+6 u v+6 v^{2}\right) .
\end{aligned}
$$

We note that $\lambda_{a, b}\left(2 T_{2,1} ; \mathbf{x}_{1}, \mathbf{x}_{2}\right)=1$ for any $\langle a, b\rangle \in C_{0}\left(T_{2,1}\langle 6,3\rangle\right)$, since $\mathbf{x}_{1}$ and $\mathbf{x}_{2}$ are linealy independent and $\mathbf{x}_{1}, \mathbf{x}_{2}$ and $\mathbf{y}$ with $\left(\mathbf{x}_{1}, \mathbf{y}\right)=a,\left(\mathbf{x}_{2}, \mathbf{y}\right)=b$ are linearly dependent.

The general formula (4.1) is specified to

$$
a\left(\left(T_{2,1},\{a / 2, b / 2\}, 3\right), \mathscr{L}_{48}\right)=\sum_{\substack{\mathbf{x}_{1}, \mathbf{x}_{2} \in \Lambda_{6} \\\left[\mathbf{x}_{1}, \mathbf{x}_{2}\right]=2 T_{2,1}}} \lambda_{a, b}\left(2 T_{2,1} ; \mathbf{x}_{1}, \mathbf{x}_{2}\right) .
$$

Taking the sum over $\mathbf{x}_{1}, \mathbf{x}_{2} \in \lambda_{6}$ in the above equation we have the equation (for a spacing problem we use $\lambda_{a, b}\left(2 T_{2,1}\right)$ instead of $\lambda_{a, b}\left(2 T_{2,1} ; \mathbf{x}_{1}, \mathbf{x}_{2}\right)$ ):

$$
\begin{aligned}
& \sum_{\substack{\mathbf{x}_{1}, \mathbf{x}_{2} \in \Lambda_{6} \\
\left[\mathbf{x}_{1}, \mathbf{x}_{2}\right]=2 T_{2,1}}} \sum_{\langle a, b\rangle \in C_{78}\left(T_{2,1},\langle 1,1\rangle\right)} \lambda_{a, b}\left(2 T_{2,1}\right)(a u+b v)^{2} \\
& +\sum_{\substack{\mathbf{x}_{1}, \mathbf{x}_{2} \in \Lambda_{6} \\
\left[\mathbf{x}_{1}, \mathbf{x}_{2}\right]=2 T_{2,1}}} \sum_{\langle a, b\rangle \in C_{72}\left(T_{2,1},\langle 2,1\rangle\right)} \lambda_{a, b}\left(2 T_{2,1}\right)(a u+b v)^{2} \\
& +\sum_{\substack{\mathbf{x}_{1}, \mathbf{x}_{2} \in \Lambda_{6} \\
\left[\mathbf{x}_{1}, \mathbf{x}_{2}\right]=2 T_{2,1}}} \sum_{\langle a, b\rangle \in C_{69}\left(T_{2,1},\langle 2,2\rangle\right)} \lambda_{a, b}\left(2 T_{2,1}\right)(a u+b v)^{2} \\
& +\sum_{\substack{\mathbf{x}_{1}, \mathbf{x}_{2} \in \Lambda_{6} \\
\left[\mathbf{x}_{1}, \mathbf{x}_{2}\right]=2 T_{2,1}}} \sum_{\langle a, b\rangle \in C_{60}\left(T_{2,1},\langle 3,2\rangle\right)} \lambda_{a, b}\left(2 T_{2,1}\right)(a u+b v)^{2} \\
& +\sum_{\substack{\mathbf{x}_{1}, \mathbf{x}_{2} \in \Lambda_{6} \\
\left[\mathbf{x}_{1}, \mathbf{x}_{2}\right]=2 T_{2,1}}} \sum_{\langle a, b\rangle \in C_{54}\left(T_{2,1},\langle 3,3\rangle\right)} \lambda_{a, b}\left(2 T_{2,1}\right)(a u+b v)^{2}
\end{aligned}
$$

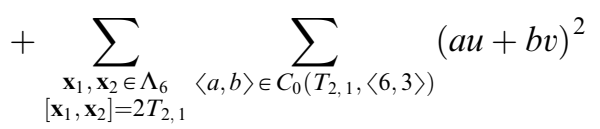

$$
\begin{aligned}
& =6552000\left(6 u^{2}+6 u v+6 v^{2}\right) \sum_{\substack{\mathbf{x}_{1}, \mathbf{x}_{2} \in \Lambda_{6} \\
\left[\mathbf{x}_{1}, \mathbf{x}_{2}\right]=2 T_{2,1}}} 1 .
\end{aligned}
$$


We note that

$$
\begin{aligned}
& \sum_{\substack{\mathbf{x}_{1}, \mathbf{x}_{2} \in \Lambda_{6} \\
\left[\mathbf{x}_{1}, \mathbf{x}_{2}\right]=2 T_{2,1}}} \sum_{\langle a, b\rangle \in C_{78}\left(T_{2,1},\langle 1,1\rangle\right)} \lambda_{a, b}\left(2 T_{2,1}\right)(a u+b v)^{2} \\
& =\sum_{\langle a, b\rangle \in C_{78}\left(T_{2,1},\langle 1,1\rangle\right)} \sum_{\substack{\mathbf{x}_{1}, \mathbf{x}_{2} \in \Lambda_{6} \\
\left[\mathbf{x}_{1}, \mathbf{x}_{2}\right]=2 T_{2,1}}} \lambda_{a, b}\left(2 T_{2,1}\right)(a u+b v)^{2} \\
& =\sum_{\langle a, b\rangle \in C_{78}\left(T_{2,1},\langle 1,1\rangle\right)} a\left(\left(T_{2,1},\{a / 2, b / 2\}, 3\right), \mathscr{L}_{48}\right)(a u+b v)^{2} \\
& =a\left(\left(T_{2,1},\{1 / 2,1 / 2\}, 3\right), \mathscr{L}_{48}\right) \sum_{\langle a, b\rangle \in C_{78}\left(T_{2,1},\langle 1,1\rangle\right)}(a u+b v)^{2}
\end{aligned}
$$

The last transition is supported by the fact that for each meber $\langle a, b\rangle$ of $C_{78}\left(T_{2,1},\langle 1,1\rangle\right)$ the matrix $\left(T_{2,1},\{a / 2, b / 2\}, 3\right)$ is integarally equivalent and by Proposition 2.4. Likewise we can show that

$$
\begin{aligned}
& \sum_{\substack{\mathbf{x}_{1}, \mathbf{x}_{2} \in \Lambda_{6} \\
\left[\mathbf{x}_{1}, \mathbf{x}_{2}\right]=2 T_{2,1}}} \sum_{\langle a, b\rangle \in C_{72}\left(T_{2,1},\langle 2,1\rangle\right)} \lambda_{a, b}\left(2 T_{2,1}\right)(a u+b v)^{2} \\
& =\sum_{\langle a, b\rangle \in C_{78}\left(T_{2,1},\langle 1,1\rangle\right)} \sum_{\substack{\mathbf{x}_{1}, \mathbf{x}_{2} \in \Lambda_{6} \\
\left[\mathbf{x}_{1}, \mathbf{x}_{2}\right]=2 T_{2,1}}} \lambda_{a, b}\left(2 T_{2,1}\right)(a u+b v)^{2} \\
& =a\left(\left(T_{2,1},\{1,1 / 2\}, 3\right), \mathscr{L}_{48}\right) \sum_{\langle a, b\rangle \in C_{72}\left(T_{2,1},\langle 2,1\rangle\right)}(a u+b v)^{2}
\end{aligned}
$$

and etc. To save space we will use the shorthand: $A_{7}=a\left(\left(T_{2,1},\{1 / 2,1 / 2\}, 3\right)\right.$, $\left.\mathscr{L}_{48}\right), \ldots, A_{1}=a\left(\left(T_{2,1},\{3 / 3,3 / 2\}, 3\right), \mathscr{L}_{48}\right)$. (Conf. Table 2.) We have the equation

$$
\begin{aligned}
& A_{7} \sum_{\langle a, b\rangle \in C_{78}\left(T_{2,1},\langle 1,1\rangle\right)}(a u+b v)^{2}+A_{5} \sum_{\langle a, b\rangle \in C_{72}\left(T_{2,1},\langle 2,1\rangle\right)}(a u+b v)^{2} \\
& \quad+A_{4} \sum_{\langle a, b\rangle \in C_{69}\left(T_{2,1},\langle 2,2\rangle\right)}(a u+b v)^{2}+A_{2} \sum_{\langle a, b\rangle \in C_{60}\left(T_{2,1},\langle 3,2\rangle\right)}(a u+b v)^{2} \\
& \quad+A_{1} \sum_{\langle a, b\rangle \in C_{54}\left(T_{2,1},\langle 3,3\rangle\right)}(a u+b v)^{2} \\
& \quad+a\left(T_{2,1}, \mathscr{L}_{48}\right) \sum_{\langle a, b\rangle \in C_{0}\left(T_{2,1},\langle 6,3\rangle\right)}(a u+b v)^{2} \\
&= 6552000\left(6 u^{2}+6 u v+6 v^{2}\right) a\left(T_{2,1}, \mathscr{L}_{48}\right) .
\end{aligned}
$$


The above equation is a polynomial identity with respect to the variables $u, v$ and we hve linear constraints concerning $A_{7}, \ldots, A_{1}$.

Similarly from the formula $(2.2) \sim(2.4)$ we have the equations:

$$
\begin{aligned}
& A_{7} \sum_{\langle a, b\rangle \in C_{78}\left(T_{2,1},\langle 1,1\rangle\right)}(a u+b v)^{4}+A_{5} \sum_{\langle a, b\rangle \in C_{72}\left(T_{2,1},\langle 2,1\rangle\right)}(a u+b v)^{4} \\
& +A_{4} \sum_{\langle a, b\rangle \in C_{69}\left(T_{2,1},\langle 2,2\rangle\right)}(a u+b v)^{4}+A_{2} \sum_{\langle a, b\rangle \in C_{60}\left(T_{2,1},\langle 3,2\rangle\right)}(a u+b v)^{4} \\
& +A_{1} \sum_{\langle a, b\rangle \in C_{54}\left(T_{2,1},\langle 3,3\rangle\right)}(a u+b v)^{4} \\
& +a\left(T_{2,1}, \mathscr{L}_{48}\right) \sum_{\langle a, b\rangle \in C_{0}\left(T_{2,1},\langle 6,3\rangle\right)}(a u+b v)^{4} \\
& =2358720\left(6 u^{2}+6 u v+6 v^{2}\right)^{2} a\left(T_{2,1}, \mathscr{L}_{48}\right), \\
& A_{7} \sum_{\langle a, b\rangle \in C_{78}\left(T_{2,1},\langle 1,1\rangle\right)}(a u+b v)^{6}+A_{5} \sum_{\langle a, b\rangle \in C_{72}\left(T_{2,1},\langle 2,1\rangle\right)}(a u+b v)^{6} \\
& +A_{4} \sum_{\langle a, b\rangle \in C_{69}\left(T_{2,1},\langle 2,2\rangle\right)}(a u+b v)^{6}+A_{2} \sum_{\langle a, b\rangle \in C_{60}\left(T_{2,1},\langle 3,2\rangle\right)}(a u+b v)^{6} \\
& +A_{1} \sum_{\langle a, b\rangle \in C_{54}\left(T_{2,1},\langle 3,3\rangle\right)}(a u+b v)^{6} \\
& +a\left(T_{2,1}, \mathscr{L}_{48}\right) \sum_{\langle a, b\rangle \in C_{0}\left(T_{2,1},\langle 6,3\rangle\right)}(a u+b v)^{6} \\
& =1360800\left(6 u^{2}+6 u v+6 v^{2}\right)^{3} a\left(T_{2,1}, \mathscr{L}_{48}\right), \\
& A_{7} \sum_{\langle a, b\rangle \in C_{78}\left(T_{2,1},\langle 1,1\rangle\right)}(a u+b v)^{8}+A_{5} \sum_{\langle a, b\rangle \in C_{72}\left(T_{2,1},\langle 2,1\rangle\right)}(a u+b v)^{8} \\
& +A_{4} \sum_{\langle a, b\rangle \in C_{69}\left(T_{2,1},\langle 2,2\rangle\right)}(a u+b v)^{8}+A_{2} \sum_{\langle a, b\rangle \in C_{60}\left(T_{2,1},\langle 3,2\rangle\right)}(a u+b v)^{8} \\
& +A_{1} \sum_{\langle a, b\rangle \in C_{54}\left(T_{2,1},\langle 3,3\rangle\right)}(a u+b v)^{8} \\
& +a\left(T_{2,1}, \mathscr{L}_{48}\right) \sum_{\langle a, b\rangle \in C_{0}\left(T_{2,1},\langle 6,3\rangle\right)}(a u+b v)^{8} \\
& =\left(6 u^{2}+6 u v+6 v^{2}\right)^{4} a\left(T_{2,1}, \mathscr{L}_{48}\right) .
\end{aligned}
$$

The above four polynomial identities are enough to solve $A_{1}, \ldots, A_{7}$. We have

$$
\begin{aligned}
& A_{7}=5365440 a\left(T_{2,1}, \mathscr{L}_{48}\right), \\
& A_{5}=922185 a\left(T_{2,1}, \mathscr{L}_{48}\right), \\
& A_{4}=361584 a\left(T_{2,1}, \mathscr{L}_{48}\right),
\end{aligned}
$$




$$
\begin{aligned}
A_{2} & =16767 a\left(T_{2,1}, \mathscr{L}_{48}\right), \\
& =a\left(\left(T_{2,1},\{3 / 2,1 / 2\}, 3\right), \mathscr{L}_{48}\right) \\
& =a\left(T_{3,2}, \mathscr{L}_{48}\right)=32384199085056000
\end{aligned}
$$

(we will use this value at Proposition 5.4),

$$
\begin{aligned}
A_{1} & =1656 a\left(T_{2,1}, \mathscr{L}_{48}\right) \\
& =a\left(\left(T_{2,1},\{3 / 2,3 / 2\}, 3\right), \mathscr{L}_{48}\right) \\
& =a\left(T_{3,1}, \mathscr{L}_{48}\right)=3198439415808000
\end{aligned}
$$

(we will use this value at Proposition 5.3).

Here $a\left(T_{2,1}, \mathscr{L}_{48}\right)=1931424768000$. As to $a\left(\left(T_{2,1},\{0,0\}, 3\right), \mathscr{L}_{48}\right)$ we use the formula (2.0). The lefthand side of (2.0) is slightly different from the formulas (2.1) (2.5). Skipping the earlier equations we reach

$$
\begin{aligned}
a\left(\left(T_{2,1},\right.\right. & \left.\{0,0\}, 3), \mathscr{L}_{48}\right) c_{81}\left(T_{2,1}\right)+A_{7} \cdot c_{78}\left(T_{2,1}\right)+A_{5} \cdot c_{72}\left(T_{2,1}\right)+A_{4} \cdot c_{64}\left(T_{2,1}\right) \\
& +A_{2} \cdot c_{60}\left(T_{2,1}\right)+A_{1} \cdot c_{54}\left(T_{2,1}\right)+a\left(T_{2,1}, \mathscr{L}_{48}\right) \cdot c_{0}\left(T_{2,1}\right) \\
= & a\left(T_{2,1}, \mathscr{L}_{48}\right) \cdot 52416000 .
\end{aligned}
$$

From this we have $\left.a\left(\left(T_{2,1},\{0,0\}, 3\right), \mathscr{L}_{48}\right)\right)=23775066324172800000$.

\subsubsection{The Second Case (a Summary)}

For a fixed pair $\mathbf{x}_{1}, \mathbf{x}_{2} \in \Lambda_{6}$ which satisfy $\left[\mathbf{x}_{1}, \mathbf{x}_{2}\right]=2 T_{2,2}$, we set

$$
\lambda_{a, b}\left(2 T_{2,2} ; \mathbf{x}_{1}, \mathbf{x}_{2}\right)=\left|\left\{\mathbf{z} \in \Lambda_{6} \mid\left(\mathbf{x}_{1}, \mathbf{z}\right)=a,\left(\mathbf{x}_{2}, \mathbf{z}\right)=b\right\}\right| .
$$

We take $\boldsymbol{\alpha}=u \mathbf{x}_{1}+v \mathbf{x}_{2}$, where $u$ and $v$ are real independent variables, then we see that for $\mathbf{y} \in \Lambda_{6}$

$$
(\mathbf{y}, \boldsymbol{\alpha})=a u+b v
$$

and

$$
(\boldsymbol{\alpha}, \boldsymbol{\alpha})=6 u^{2}+4 u v+6 v^{2}
$$

Applying the formulas $(2.1) \sim(2.5)$ in this settings we have 


$$
\begin{aligned}
A_{14} \sum_{\langle a, b\rangle \in C_{93}\left(T_{2,2},\langle 1,0\rangle\right)}(a u+b v)^{2 k} & \\
& +A_{13} \sum_{\langle a, b\rangle \in C_{92}\left(T_{2,2},\langle 1,1\rangle\right)}(a u+b v)^{2 k} \\
& +A_{12} \sum_{\langle a, b\rangle \in C_{88}\left(T_{2,2},\langle 1,-1\rangle\right)}(a u+b v)^{2 k} \\
& +A_{11} \sum_{\langle a, b\rangle \in C_{85}\left(T_{2,2},\langle 2,1\rangle\right)}(a u+b v)^{2 k} \\
& +A_{10} \sum_{\langle a, b\rangle \in C_{84}\left(T_{2,2},\langle 2,0\rangle\right)}(a u+b v)^{2 k} \\
& +A_{8} \sum_{\langle a, b\rangle \in C_{80}\left(T_{2,2},\langle 2,2\rangle\right)}(a u+b v)^{2 k} \\
& +A_{6} \sum_{\langle a, b\rangle \in C_{77}\left(T_{2,2},\langle 2,-1\rangle\right)}(a u+b v)^{2 k} \\
& +A_{5} \sum_{\langle a, b\rangle \in C_{72}\left(T_{2,2},\langle 3,1\rangle\right)}(a u+b v)^{2 k} \\
& +A_{4} \sum_{\langle a, b\rangle \in C_{69}\left(T_{2,2},\langle 3,2\rangle\right)}(a u+b v)^{2 k} \\
& +A_{3} \sum_{\langle a, b\rangle \in C_{64}\left(T_{2,2},\langle 2,-2\rangle\right)}(a u+b v)^{2 k} \\
& +A_{2} \sum_{\langle a, b\rangle \in C_{60}\left(T_{2,2},\langle 3,3\rangle\right)}(a u+b v)^{2 k} \\
& +a\left(T_{2,2}, \mathscr{L}_{48}\right) \sum_{\langle a, b\rangle \in C_{0}\left(T_{2,2},\langle 6,2\rangle\right)}(a u+b v)^{2 k} \\
& \rho_{k}\left(6 u^{2}+4 u v+6 v^{2}\right)^{k} a\left(T_{2,2}, \mathscr{L}_{48}\right) \\
&
\end{aligned}
$$

where

$$
\rho_{k}= \begin{cases}6552000 & \text { if } k=1 \\ 2358720 & \text { if } k=2, \\ 1360800 & \text { if } k=3 \\ 1058400 & \text { if } k=4 \\ 1020600 & \text { if } k=5\end{cases}
$$

Again we have linear constraints on $A_{14} \ldots, A_{2}$ and we solve them

$$
\begin{aligned}
& A_{14}=5623040 a\left(T_{2,2}, \mathscr{L}_{48}\right), \\
& A_{13}=4432560 a\left(T_{2,2}, \mathscr{L}_{48}\right), \\
& A_{12}=1667040 a\left(T_{2,2}, \mathscr{L}_{48}\right), \\
& A_{11}=777216 a\left(T_{2,2}, \mathscr{L}_{48}\right),
\end{aligned}
$$




$$
\begin{aligned}
& A_{10}=599104 a\left(T_{2,2}, \mathscr{L}_{48}\right), \\
& A_{8}=204792 a\left(T_{2,2}, \mathscr{L}_{48}\right), \\
& A_{6}=88320 a\left(T_{2,2}, \mathscr{L}_{48}\right), \\
& A_{5}=20240 a\left(T_{2,2}, \mathscr{L}_{48}\right), \\
& A_{4}=7936 a\left(T_{2,2}, \mathscr{L}_{48}\right), \\
& A_{3}=1518 a\left(T_{2,2}, \mathscr{L}_{48}\right), \\
& A_{2}=368 a\left(T_{2,2}, \mathscr{L}_{48}\right) .
\end{aligned}
$$

Here $a\left(T_{2,2}, \mathscr{L}_{48}\right)=88000540992000$. As for $\left.a\left(\left(T_{2,2},\{0,0\}, 3\right), \mathscr{L}_{48}\right)\right)$ by taking a similar way to the last part of Section 4.3 .1 we obtain

$$
\left.a\left(\left(T_{2,2},\{0,0\}, 3\right), \mathscr{L}_{48}\right)\right)=995004516888345600000 .
$$

REMARK 4. In our present computations we do not refer the formula (2.6), since this formula gives us no new information other than the ones from the formulas $(2.1) \sim(2.5)$. The same comment is also applied to the later sections.

\subsubsection{The Third Case (a Summary)}

For a fixed pair $\mathbf{x}_{1}, \mathbf{x}_{2} \in \Lambda_{6}$ which satisfy $\left[\mathbf{x}_{1}, \mathbf{x}_{2}\right]=2 T_{2,3}$, we set

$$
\lambda_{a, b}\left(2 T_{2,3} ; \mathbf{x}_{1}, \mathbf{x}_{2}\right)=\left|\left\{\mathbf{z} \in \Lambda_{6} \mid\left(\mathbf{x}_{1}, \mathbf{z}\right)=a,\left(\mathbf{x}_{2}, \mathbf{z}\right)=b\right\}\right| .
$$

We take $\boldsymbol{\alpha}=u \mathbf{x}_{1}+v \mathbf{x}_{2}$, where $u$ and $v$ are real independent variables, then we see that for $\mathbf{y} \in \Lambda_{6}$

$$
(\mathbf{y}, \boldsymbol{\alpha})=a u+b v
$$

and

$$
(\boldsymbol{\alpha}, \boldsymbol{\alpha})=6 u^{2}+2 u v+6 v^{2} .
$$

Applying the formulas $(2.1) \sim(2.5)$ in this settings we have

$$
\begin{aligned}
A_{18} \sum_{\langle a, b\rangle \in C_{102}\left(T_{2,3},\langle 1,0\rangle\right)}(a u+b v)^{2 k} \\
\quad+A_{17} \sum_{\langle a, b\rangle \in C_{100}\left(T_{2,3},\langle 1,1\rangle\right)}(a u+b v)^{2 k} \\
+A_{16} \sum_{\langle a, b\rangle \in C_{98}\left(T_{2,3},\langle 1,-1\rangle\right)}(a u+b v)^{2 k} \\
\quad+A_{14} \sum_{\langle a, b\rangle \in C_{93}\left(T_{2,3},\langle 2,0\rangle\right)}(a u+b v)^{2 k}
\end{aligned}
$$




$$
\begin{aligned}
& +A_{13} \sum_{\langle a, b\rangle \in C_{92}\left(T_{2,3},\langle 2,1\rangle\right)}(a u+b v)^{2 k} \\
& +A_{12} \sum_{\langle a, b\rangle \in C_{88}\left(T_{2,3},\langle 2,-1\rangle\right)}(a u+b v)^{2 k} \\
& +A_{11} \sum_{\langle a, b\rangle \in C_{85}\left(T_{2,3},\langle 2,2\rangle\right)}(a u+b v)^{2 k} \\
& +A_{7} \sum_{\langle a, b\rangle \in C_{78}\left(T_{2,3},\langle 3,1\rangle\right)}(a u+b v)^{2 k} \\
& +A_{6} \sum_{\langle a, b\rangle \in C_{77}\left(T_{2,3},\langle 2,-2\rangle\right)}(a u+b v)^{2 k} \\
& +A_{5} \sum_{\langle a, b\rangle \in C_{72}\left(T_{2,3},\langle 3,2\rangle\right)}(a u+b v)^{2 k} \\
& +A_{2} \sum_{\langle a, b\rangle \in C_{60}\left(T_{2,3},\langle 3,3\rangle\right)}(a u+b v)^{2 k} \\
& +a\left(T_{2,3}, \mathscr{L}_{48}\right) \sum_{\langle a, b\rangle \in C_{0}\left(T_{2,3},\langle 6,1\rangle\right)}(a u+b v)^{2 k} \\
& =\rho_{k}\left(6 u^{2}+2 u v+6 v^{2}\right)^{k} a\left(T_{2,3}, \mathscr{L}_{48}\right), \quad k=1, \ldots, 5 .
\end{aligned}
$$

We have

$$
\begin{aligned}
& A_{18}=5713400 a\left(T_{2,3}, \mathscr{L}_{48}\right), \\
& A_{17}=3695433 a\left(T_{2,3}, \mathscr{L}_{48}\right), \\
& A_{16}=2369400 a\left(T_{2,3}, \mathscr{L}_{48}\right), \\
& A_{14}=748720 a\left(T_{2,3}, \mathscr{L}_{48}\right), \\
& A_{13}=590205 a\left(T_{2,3}, \mathscr{L}_{48}\right), \\
& A_{12}=221970 a\left(T_{2,3}, \mathscr{L}_{48}\right), \\
& A_{11}=103488 a\left(T_{2,3}, \mathscr{L}_{48}\right), \\
& A_{7}=15680 a\left(T_{2,3}, \mathscr{L}_{48}\right), \\
& A_{6}=11760 a\left(T_{2,3}, \mathscr{L}_{48}\right), \\
& A_{5}=2695 a\left(T_{2,3}, \mathscr{L}_{48}\right), \\
& A_{2}=49 a\left(T_{2,3}, \mathscr{L}_{48}\right) .
\end{aligned}
$$

Here $a\left(T_{2,3}, \mathscr{L}_{48}\right)=660902022144000$. As for the value $a\left(\left(T_{2,3},\{0,0\}, 3\right), \mathscr{L}_{48}\right)$ a similar effort to Sections 4.3 .1 and 4.3 .2 works and we have

$$
a\left(\left(T_{2,3},\{0,0\}, 3\right), \mathscr{L}_{48}\right)=7145249686126755840000 .
$$




\subsubsection{The Fourth Case (a Summary)}

For a fixed pair $\mathbf{x}_{1}, \mathbf{x}_{2} \in \Lambda_{6}$ which satisfy $\left[\mathbf{x}_{1}, \mathbf{x}_{2}\right]=2 T_{2,4}$, we set

$$
\lambda_{a, b}\left(2 T_{2,4} ; \mathbf{x}_{1}, \mathbf{x}_{2}\right)=\left|\left\{\mathbf{z} \in \Lambda_{6} \mid\left(\mathbf{x}_{1}, \mathbf{z}\right)=a,\left(\mathbf{x}_{2}, \mathbf{z}\right)=b\right\}\right| .
$$

We take $\boldsymbol{a}=u \mathbf{x}_{1}+v \mathbf{x}_{2}$, where $u$ and $v$ are real independent variables, then we see that for $\mathbf{y} \in \Lambda_{6}$

$$
(\mathbf{y}, \boldsymbol{\alpha})=a u+b v
$$

and

$$
(\boldsymbol{\alpha}, \boldsymbol{\alpha})=6 u^{2}+6 v^{2} .
$$

Applying the formulas $(2.1) \sim(2.5)$ in this settings we have

$$
\begin{aligned}
A_{19} \sum_{\langle a, b\rangle \in C_{105}\left(T_{2,4},\langle 1,0\rangle\right)}(a u+b v)^{2 k}+A_{18} \sum_{\langle a, b\rangle \in C_{102}\left(T_{2,4},\langle 1,1\rangle\right)}(a u+b v)^{2 k} \\
\quad+A_{15} \sum_{\langle a, b\rangle \in C_{96}\left(T_{2,4},\langle 2,0\rangle\right)}(a u+b v)^{2 k}+A_{14} \sum_{\langle a, b\rangle \in C_{93}\left(T_{2,4},\langle 2,1\rangle\right)}(a u+b v)^{2 k} \\
\quad+A_{10} \sum_{\langle a, b\rangle \in C_{84}\left(T_{2,4},\langle 2,2\rangle\right)}(a u+b v)^{2 k}+A_{9} \sum_{\langle a, b\rangle \in C_{81}\left(T_{2,4},\langle 3,0\rangle\right)}(a u+b v)^{2 k} \\
\quad+A_{7} \sum_{\langle a, b\rangle \in C_{78}\left(T_{2,4},\langle 3,1\rangle\right)}(a u+b v)^{2 k}+A_{4} \sum_{\langle a, b\rangle \in C_{69}\left(T_{2,4},\langle 3,2\rangle\right)}(a u+b v)^{2 k} \\
\quad+A_{1} \sum_{\langle a, b\rangle \in C_{54}\left(T_{2,4},\langle 3,3\rangle\right)}(a u+b v)^{2 k} \\
\quad+a\left(T_{2,4}, \mathscr{L}_{48}\right) \sum_{\langle a, b\rangle \in C_{0}\left(T_{2,4},\langle 6,0\rangle\right)}(a u+b v)^{2 k} \\
=\rho_{k}\left(6 u^{2}+6 v^{2}\right)^{k} a\left(T_{2,4}, \mathscr{L}_{48}\right), \quad k=1, \ldots, 5 .
\end{aligned}
$$

From these we have linear equations on $A_{19}, \ldots, A_{1}$. This time we can not solve the linear equations. We only obtain relations among $A_{19}, \ldots, A_{1}$.

$$
\begin{aligned}
& A_{19}=5736384 a\left(T_{2,4}, \mathscr{L}_{48}\right)-300 A_{1}, \\
& A_{18}=3030480 a\left(T_{2,4}, \mathscr{L}_{48}\right)+225 A_{1}, \\
& A_{15}=798399 a\left(T_{2,4}, \mathscr{L}_{48}\right)+120 A_{1}, \\
& A_{14}=397440 a\left(T_{2,4}, \mathscr{L}_{48}\right)-90 A_{1}, \\
& A_{10}=42228 a\left(T_{2,4}, \mathscr{L}_{48}\right)+36 A_{1}, \\
& A_{9}=19136 a\left(T_{2,4}, \mathscr{L}_{48}\right)-20 A_{1},
\end{aligned}
$$




$$
\begin{aligned}
& A_{7}=8280 a\left(T_{2,4}, \mathscr{L}_{48}\right)+15 A_{1}, \\
& A_{4}=576 a\left(T_{2,4}, \mathscr{L}_{48}\right)-6 A_{1} .
\end{aligned}
$$

Here $a\left(T_{2,4}, \mathscr{L}_{48}\right)=1245768975360000$, and $A_{1}$ is computed in the previous sections. $A_{1}=3198439415808000$. The value $a\left(\left(T_{2,4},\{0,0\}, 3\right), \mathscr{L}_{48}\right)$ can be deduced from $A_{19}, \ldots, A_{1}$ likewisely, and we have

$$
a\left(\left(T_{2,4},\{0,0\}, 3\right), \mathscr{L}_{48}\right)=13280080376405606400000 .
$$

\subsubsection{A Table}

Table 3. Fourier coefficints of Siegel theta series of degree 3 for the 48 dimensional extremal lattice

\begin{tabular}{|c|r|l|r|}
\hline num & \multicolumn{1}{|c|}{$D$} & reduced $T$ & \multicolumn{1}{|c|}{$a\left(T, \mathcal{L}_{48}\right)$} \\
\hline 1 & $* 54$ & $(3,3,3,3,3,0)$ & 3198439415808000 \\
2 & 60 & $(3,3,3,3,2,-1)$ & 32384199085056000 \\
3 & 64 & $(3,3,3,2,2,-2)$ & 133584821225856000 \\
4 & 69 & $(3,3,3,3,2,0)$ & 698372293312512000 \\
5 & 72 & $(3,3,3,3,1,-1)$ & 1781130949678080000 \\
6 & 77 & $(3,3,3,2,2,-1)$ & 7772207780413440000 \\
7 & 78 & $(3,3,3,3,1,0)$ & 10362943707217920000 \\
8 & 80 & $(3,3,3,2,2,2)$ & 18021806790833664000 \\
9 & $* 81$ & $(3,3,3,3,0,0)$ & 23775066324172800000 \\
10 & 84 & $(3,3,3,2,2,0)$ & 52721476110471168000 \\
11 & 85 & $(3,3,3,2,2,1)$ & 68395428467638272000 \\
12 & 88 & $(3,3,3,2,1,-1)$ & 146700421855303680000 \\
13 & 92 & $(3,3,3,2,1,1)$ & 390067677979499520000 \\
14 & 93 & $(3,3,3,2,1,0)$ & 494830562019655680000 \\
15 & 96 & $(3,3,3,2,0,0)$ & 995004516888345600000 \\
16 & 98 & $(3,3,3,1,1,-1)$ & 1565941251267993600000 \\
17 & 100 & $(3,3,3,1,1,1)$ & 2442319142397668352000 \\
18 & 102 & $(3,3,3,1,1,0)$ & 3775997613317529600000 \\
19 & 105 & $(3,3,3,1,0,0)$ & 7145249686126755840000 \\
20 & $* 108$ & $(3,3,3,0,0,0)$ & 13280080376405606400000 \\
\hline
\end{tabular}

REMARK 5. In Table 3 we only give the values of the Fourier coefficients $a\left(T, \mathscr{L}_{48}\right)$ with the restriction that the diagonal entries of $M R(T)$ are all 3. But our method enables us to compute $a\left(T, \mathscr{L}_{48}\right)$ for the indeces $T$ such that the diagonal entries of $M R(T)$ are $\geq 3$.

\section{Fourier Coefficients of Siegel Theta Series of Degree 4 for $\mathscr{L}$}

Our plan to compute the Fourier coefficients or to find the relations among the Fourier coefficients is as follows. 


\subsection{Extending the Matrices of Degree 3 to the Matrices of Degree 4}

The following result which is due to Schiemann is quite useful.

Proposition 5.1 (Schiemann [17]). Except one pair of forms two positive definite quaternary even integral quadratic forms with the same discriminant $\leq 3000$ are integrally equivalent if and only if their theta series of one variable equal.

The only exceptional pair of forms is given at the discriminant 1729.

By Proposition 5.1 we can quickly and effectively distinguish whether two quaternary quadratic forms are integrally equivalent or not.

\subsubsection{First Case}

We put

$$
2 \cdot T_{3,1}=\left(\begin{array}{lll}
6 & 3 & 3 \\
3 & 6 & 3 \\
3 & 3 & 6
\end{array}\right)
$$

For a fixed triple $\left\langle\mathbf{x}_{1}, \mathbf{x}_{2}, \mathbf{x}_{3}\right\rangle$ satisfying $\left[\mathbf{x}_{1}, \mathbf{x}_{2}, \mathbf{x}_{3}\right]=2 \cdot T_{3,1}$ we seek to find out all ordered triples $\langle a, b, c\rangle$ of integers satisfying the two conditions

(1) $\left(T_{3,1},\{a / 2, b / 2, c / 2\}, 3\right)$ is positive semi-definite,

(2) when $\left(T_{3,1},\{a / 2, b / 2, c / 2\}, 3\right)$ is reduced under the unimodular transformation

$U^{t}\left(T_{3,1},\{a / 2, b / 2, c / 2\}, 3\right) U$, the minimal value of the non-zero diagonal entries of the resulting matrix is 3 .

It is rather easy to find all triples $\langle a, b, c\rangle$ satisfying the condition (1) only. Next from all such triples we form the sets of triples with two further conditions (i) in a set each triple $\langle a, b, c\rangle$ has identical $\operatorname{det}\left(2\left(T_{3,1},\{a / 2, b / 2, c / 2\}, 3\right)\right)$ (this is called the discriminant of $\left(T_{3,1},\{a / 2, b / 2, c / 2\}, 3\right)$ ), (ii) each member of triple $\langle a, b, c\rangle$ in a set determines the unique integrally equivalent form $2\left(T_{3,1},\{a / 2, b / 2, c / 2\}, 3\right)$. To check (ii) we use Proposition 5.1. We find out that $C_{648}\left(T_{3,1},\langle 0,0,0\rangle\right)$ and

$$
\begin{aligned}
& C_{621}^{+}\left(T_{3,1},\langle 1,1,1\rangle\right)=\{\langle 1,1,1\rangle,\langle 1,0,0\rangle,\langle 0,1,0\rangle,\langle 0,0,1\rangle\} \\
& C_{612}^{+}\left(T_{3,1},\langle 1,1,0\rangle\right)=\{\langle 1,1,0\rangle,\langle 1,0,1\rangle,\langle 0,1,1\rangle\} \\
& C_{576}^{+}\left(T_{3,1},\langle 2,1,1\rangle\right)=\{\langle 2,1,1\rangle,\langle 1,2,1\rangle,\langle 1,1,2\rangle,\langle 1,0,-1\rangle, \\
&\langle 1,-1,0\rangle,\langle 0,1,-1\rangle\}
\end{aligned}
$$




$$
\begin{aligned}
& C_{549}^{+}\left(T_{3,1},\langle 2,2,1\rangle\right)=\{\langle 2,2,1\rangle,\langle 2,1,2\rangle,\langle 2,1,0\rangle,\langle 2,0,1\rangle,\langle 1,2,2\rangle, \\
& \langle 1,2,0\rangle,\langle 1,1,-1\rangle,\langle 1,0,2\rangle,\langle 1,-1,1\rangle,\langle 1,-1,-1\rangle, \\
& \langle 0,2,1\rangle,\langle 0,1,2\rangle\}, \\
& C_{540}^{+}\left(T_{3,1},\langle 2,2,2\rangle\right)=\{\langle 2,2,2\rangle,\langle 2,0,0\rangle,\langle 0,2,0\rangle,\langle 0,0,2\rangle\}, \\
& C_{504}^{+}\left(T_{3,1},\langle 2,2,0\rangle\right)=\{\langle 2,2,0\rangle,\langle 2,0,2\rangle,\langle 0,2,2\rangle\}, \\
& C_{477}^{+}\left(T_{3,1},\langle 3,2,2\rangle\right)=\{\langle 3,2,2\rangle,\langle 3,1,1\rangle,\langle 2,3,2\rangle,\langle 2,2,3\rangle,\langle 2,0,-1\rangle, \\
& \langle 2,-1,0\rangle,\langle 1,3,1\rangle,\langle 1,1,3\rangle,\langle 1,0,-2\rangle,\langle 1,-2,0\rangle, \\
& \langle 0,2,-1\rangle,\langle 0,1,-2\rangle\} \text {, } \\
& C_{468}^{+}\left(T_{3,1},\langle 3,2,1\rangle\right)=\{\langle 3,2,1\rangle,\langle 3,1,2\rangle,\langle 2,3,1\rangle,\langle 2,1,3\rangle,\langle 2,1,-1\rangle, \\
& \langle 2,-1,1\rangle,\langle 1,3,2\rangle,\langle 1,2,3\rangle,\langle 1,2,-1\rangle,\langle 1,-1,2\rangle, \\
& \langle 1,-1,-2\rangle,\langle 1,-2,-1\rangle\}, \\
& C_{432}^{+}\left(T_{3,1},\langle 3,3,2\rangle\right)=\{\langle 3,3,2\rangle,\langle 3,2,3\rangle,\langle 3,1,0\rangle,\langle 3,0,1\rangle,\langle 2,3,3\rangle, \\
& \langle 2,-1,-1\rangle,\langle 1,3,0\rangle,\langle 1,1,-2\rangle,\langle 1,0,3\rangle,\langle 1,-2,1\rangle \text {, } \\
& \langle 0,3,1\rangle,\langle 0,1,3\rangle\}, \\
& C_{405,1}^{+}\left(T_{3,1},\langle 3,3,3\rangle\right)=\{\langle 3,3,3\rangle,\langle 3,0,0\rangle,\langle 0,3,0\rangle,\langle 0,0,3\rangle\} \\
& C_{405,2}^{+}\left(T_{3,1},\langle 3,3,1\rangle\right)=\{\langle 3,3,1\rangle,\langle 3,2,0\rangle,\langle 3,1,3\rangle,\langle 3,0,2\rangle,\langle 2,3,0\rangle,\langle 2,0,3\rangle, \\
& \langle 2,-1,2\rangle,\langle 1,3,3\rangle,\langle 1,-2,-2\rangle,\langle 0,3,2\rangle,\langle 0,2,3\rangle\}, \\
& C_{324}^{+}\left(T_{3,1},\langle 3,3,0\rangle\right)=\{\langle 3,3,0\rangle,\langle 3,0,3\rangle,\langle 3,0,-1\rangle,\langle 3,-1,0\rangle,\langle 1,0,-3\rangle, \\
& \langle 1,-3,0\rangle,\langle 0,3,3\rangle,\langle 0,3,-1\rangle,\langle 0,1,-3\rangle\}, \\
& C_{0}^{+}\left(T_{3,1},\langle 6,3,3\rangle\right)=\{\langle 6,3,3\rangle,\langle 3,6,3\rangle,\langle 3,3,6\rangle,\langle 3,3,-2\rangle,\langle 3,0,-3\rangle, \\
& \langle 3,-2,3\rangle,\langle 3,-3,0\rangle,\langle 2,-3,-3\rangle,\langle 0,3,-3\rangle\} \text {. }
\end{aligned}
$$

Proposition 5.2. For a fixed triple $\mathbf{x}_{1}, \mathbf{x}_{2}, \mathbf{x}_{3} \in \Lambda_{6}$ which satisfy $\left[\mathbf{x}_{1}, \mathbf{x}_{2}, \mathbf{x}_{3}\right]=$ $2 T_{3,1}$ it can be verified that for any $\mathbf{y} \in \Lambda_{6}$ the triples of values $\langle a, b, c\rangle$ such that $\left(\mathbf{x}_{1}, \mathbf{y}\right)=a,\left(\mathbf{x}_{2}, \mathbf{y}\right)=b$ and $\left(\mathbf{x}_{3}, \mathbf{y}\right)=c$ fall in one of $C_{648}\left(T_{3,1},\langle 0,0,0\rangle\right)$, $C_{621}\left(T_{3,1},\langle 3,3,0\rangle\right), \ldots, C_{0}\left(T_{3,1},\langle 6,3,3\rangle\right)$. 


\subsubsection{Second Case}

We begin with a ternary matrix:

$$
2 \cdot T_{3,2}=\left(\begin{array}{lll}
6 & 3 & 3 \\
3 & 6 & 1 \\
3 & 1 & 6
\end{array}\right) .
$$

We explore all triples $\langle a, b, c\rangle$ which satisfy

(3) $\left(T_{3,2},\{a / 2, b / 2, c / 2\}, 3\right)$ is positive semi-definite,

(4) when $\left(T_{3,2},\{a / 2, b / 2, c / 2\}, 3\right)$ is reduced under the unimodular transformation:

$U^{t}\left(T_{3,2},\{a / 2, b / 2, c / 2\}, 3\right) U$, the minimal value of the non-zero diagonal entries of the resulting matrix is 3 .

Next from all such triples we form the sets of triples with two further conditions (i) in a set each triple $\langle a, b, c\rangle$ has identical $\operatorname{det}\left(2\left(T_{3,1},\{a / 2, b / 2, c / 2\}\right.\right.$, $3)$ ), (ii) each member of triple $\langle a, b, c\rangle$ in a set determines the unique integrally equivalent form $2\left(T_{3,2},\{a / 2, b / 2, c / 2\}, 3\right)$. We find out that $C_{720}\left(T_{3,2},\langle 0,0,0\rangle\right)=$ $\{\langle 0,0,0\rangle\}$ and

$$
\begin{aligned}
C_{693}^{+}\left(T_{3,2},\langle 0,1,0\rangle\right) & =\{\langle 0,1,0\rangle,\langle 0,0,1\rangle\}, \\
C_{688}^{+}\left(T_{3,2},\langle 1,1,0\rangle\right) & =\{\langle 1,1,0\rangle,\langle 1,0,1\rangle\}, \\
C_{685}^{+}\left(T_{3,2},\langle 1,1,1\rangle\right) & =\{\langle 1,1,1\rangle,\langle 1,0,0\rangle\}, \\
C_{672}^{+}\left(T_{3,2},\langle 0,1,-1\rangle\right) & =\{\langle 0,1,-1\rangle\}, \\
C_{660}^{+}\left(T_{3,2},\langle 0,1,1\rangle\right) & =\{\langle 0,1,1\rangle\}, \\
C_{640}^{+}\left(T_{3,2},\langle 2,1,1\rangle\right) & =\{\langle 2,1,1\rangle\}, \\
C_{637}^{+}\left(T_{3,2},\langle 1,2,0\rangle\right) & =\{\langle 1,2,0\rangle,\langle 1,1,-1\rangle,\langle 1,0,2\rangle,\langle 1,-1,1\rangle\}, \\
C_{628}^{+}\left(T_{3,2},\langle 1,2,1\rangle\right) & =\{\langle 1,2,1\rangle,\langle 1,1,2\rangle,\langle 1,0,-1\rangle,\langle 1,-1,0\rangle\}, \\
C_{613}^{+}\left(T_{3,2},\langle 2,2,1\rangle\right) & =\{\langle 2,2,1\rangle,\langle 2,1,2\rangle,\langle 2,1,0\rangle,\langle 2,0,1\rangle\}, \\
C_{612}^{+}\left(T_{3,2},\langle 0,2,0\rangle\right) & =\{\langle 0,2,0\rangle,\langle 0,0,2\rangle\}, \\
C_{597}^{+}\left(T_{3,2},\langle 0,2,-1\rangle\right) & =\{\langle 0,2,-1\rangle,\langle 0,1,-2\rangle\}, \\
C_{592}^{+}\left(T_{3,2},\langle 2,2,0\rangle\right) & =\{\langle 2,2,0\rangle,\langle 2,0,2\rangle,\langle 1,2,-1\rangle,\langle 1,-1,2\rangle\}, \\
C_{580}^{+}\left(T_{3,2},\langle 2,2,2\rangle\right) & =\{\langle 2,2,2\rangle,\langle 2,0,0\rangle\}, \\
C_{573}^{+}\left(T_{3,2},\langle 0,2,1\rangle\right) & =\{\langle 0,2,1\rangle,\langle 0,1,2\rangle\},
\end{aligned}
$$




$$
\begin{aligned}
& C_{565}^{+}\left(T_{3,2},\langle 1,2,2\rangle\right)=\{\langle 1,2,2\rangle,\langle 1,-1,-1\rangle\}, \\
& C_{532}^{+}\left(T_{3,2},\langle 2,3,1\rangle\right)=\{\langle 2,3,1\rangle,\langle 2,1,3\rangle,\langle 2,1,-1\rangle,\langle 2,-1,1\rangle,\langle 1,3,0\rangle, \\
& \langle 1,1,-2\rangle,\langle 1,0,3\rangle,\langle 1,-2,1\rangle\}, \\
& C_{528}^{+}\left(T_{3,2},\langle 3,2,1\rangle\right)=\{\langle 3,2,1\rangle,\langle 3,1,2\rangle,\langle 0,2,-2\rangle\}, \\
& C_{525}^{+}\left(T_{3,2},\langle 3,2,2\rangle\right)=\{\langle 3,2,2\rangle,\langle 3,1,1\rangle\}, \\
& C_{517}^{+}\left(T_{3,2},\langle 2,3,0\rangle\right)=\{\langle 2,3,0\rangle,\langle 2,2,-1\rangle,\langle 2,0,3\rangle,\langle 2,-1,2\rangle,\langle 1,3,1\rangle, \\
& \langle 1,1,3\rangle,\langle 1,0,-2\rangle,\langle 1,-2,0\rangle\}, \\
& C_{493}^{+}\left(T_{3,2},\langle 2,3,2\rangle\right)=\{\langle 2,3,2\rangle,\langle 2,2,3\rangle,\langle 2,0,-1\rangle,\langle 2,-1,0\rangle,\langle 1,3,-1\rangle, \\
& \langle 1,2,-2\rangle,\langle 1,-1,3\rangle,\langle 1,-2,2\rangle\}, \\
& C_{480}^{+}\left(T_{3,2},\langle 0,2,2\rangle\right)=\{\langle 0,2,2\rangle\}, \\
& C_{477}^{+}\left(T_{3,2},\langle 3,3,1\rangle\right)=\{\langle 3,3,1\rangle,\langle 3,2,0\rangle,\langle 3,1,3\rangle,\langle 3,0,2\rangle,\langle 0,3,0\rangle,\langle 0,0,3\rangle\}, \\
& C_{468}^{+}\left(T_{3,2},\langle 3,3,2\rangle\right)=\{\langle 3,3,2\rangle,\langle 3,2,3\rangle,\langle 3,1,0\rangle,\langle 3,0,1\rangle, \\
& \langle 0,3,-1\rangle,\langle 0,1,-3\rangle\}, \\
& C_{448}^{+}\left(T_{3,2},\langle 2,3,-1\rangle\right)=\{\langle 2,3,-1\rangle,\langle 2,-1,3\rangle,\langle 1,3,2\rangle,\langle 1,2,3\rangle, \\
& \langle 1,-1,-2\rangle,\langle 1,-2,-1\rangle\}, \\
& C_{432}^{+}\left(T_{3,2},\langle 3,3,0\rangle\right)=\{\langle 3,3,0\rangle,\langle 3,0,3\rangle,\langle 0,3,1\rangle,\langle 0,1,3\rangle\}, \\
& C_{405}^{+}\left(T_{3,2},\langle 3,3,3\rangle\right)=\{\langle 3,3,3\rangle,\langle 3,0,0\rangle,\langle 0,3,-2\rangle,\langle 0,2,-3\rangle\}, \\
& C_{400}^{+}\left(T_{3,2},\langle 2,3,3\rangle\right)=\{\langle 2,3,3\rangle,\langle 2,-1,-1\rangle,\langle 1,3,-2\rangle,\langle 1,-2,3\rangle\}, \\
& C_{0}^{+}\left(T_{3,2},\langle 6,3,3\rangle\right)=\{\langle 6,3,3\rangle,\langle 3,6,1\rangle,\langle 3,2,-3\rangle,\langle 3,1,6\rangle,\langle 3,-3,2\rangle\},
\end{aligned}
$$

\subsubsection{An Assembled Table of Quaternary Quadratic Forms}

Table 4. Table of extended quaternary quadratic forms and its reduced forms

\begin{tabular}{|c|l|}
\hline extended quaternary forms & reduced quaternary forms \\
\hline$C_{324}\left(T_{3,1}\right)$ & $(3,3,3,3,0,0,0,3,3,3)$ \\
\hline$C_{400}\left(T_{3,2}\right)$ & $(3,3,3,3,2,2,2,-3,-3,1)$ \\
\hline$C_{405,1}\left(T_{3,1}\right)$ & $(3,3,3,3,3,0,0,3,0,3)$ \\
\hline
\end{tabular}


Table 4 (continued)

\begin{tabular}{|c|c|}
\hline extended quaternary forms & reduced quaternary forms \\
\hline$C_{405}\left(T_{3,2}\right), C_{405,2}\left(T_{3,1}\right)$ & $(3,3,3,3,1,0,0,3,3,3)$ \\
\hline$C_{432}\left(T_{3,1}\right), C_{432}\left(T_{3,2}\right)$ & $(3,3,3,3,3,1,0,1,3,3)$ \\
\hline$C_{448}\left(T_{3,2}\right)$ & $(3,3,3,3,2,2,-2,1,1,3)$ \\
\hline$C_{468}\left(T_{3,1}\right), C_{468}\left(T_{3,2}\right)$ & $(3,3,3,3,1,1,0,3,3,3)$ \\
\hline$C_{477}\left(T_{3,1}\right), C_{477}\left(T_{3,2}\right)$ & $(3,3,3,3,2,1,0,0,3,3)$ \\
\hline$C_{480}\left(T_{3,2}\right)$ & $(3,3,3,3,2,0,0,2,-2,-3)$ \\
\hline$C_{493}\left(T_{3,2}\right)$ & $(3,3,3,3,3,2,0,1,2,3)$ \\
\hline$C_{504}\left(T_{3,1}\right)$ & $(3,3,3,3,3,2,0,3,0,0)$ \\
\hline$C_{517}\left(T_{3,2}\right)$ & $(3,3,3,3,3,2,-1,0,2,1)$ \\
\hline$C_{525}\left(T_{3,2}\right)$ & $(3,3,3,3,3,2,-1,2,-1,1)$ \\
\hline$C_{528}^{(1)}\left(T_{3,2}\right)$ & $(3,3,3,3,3,2,-1,2,2,2)$ \\
\hline$C_{528}^{(2)}\left(T_{3,2}\right)$ & $(3,3,3,3,3,2,-1,-1,2,-1)$ \\
\hline$C_{532}\left(T_{3,2}\right)$ & $(3,3,3,3,3,1,-1,-1,2,1)$ \\
\hline$C_{540}\left(T_{3,1}\right)$ & $(3,3,3,3,2,0,0,0,3,3)$ \\
\hline$C_{549}\left(T_{3,1}\right)$ & $(3,3,3,3,3,2,0,3,0,1)$ \\
\hline$C_{565}\left(T_{3,2}\right)$ & $(3,3,3,3,2,2,-1,3,3,1)$ \\
\hline$C_{573}\left(T_{3,2}\right)$ & $(3,3,3,3,2,2,-1,3,3,0)$ \\
\hline$C_{576}\left(T_{3,1}\right)$ & $(3,3,3,3,3,1,-1,3,0,1)$ \\
\hline$C_{580}\left(T_{3,2}\right)$ & $(3,3,3,3,3,2,-1,0,-2,2)$ \\
\hline$C_{592}^{(1)}\left(T_{3,2}\right)$ & $(3,3,3,3,3,2,0,-1,2,0)$ \\
\hline$C_{592}^{(2)}\left(T_{3,1}\right)$ & $(3,3,3,3,3,2,-1,1,2,1)$ \\
\hline$C_{597}\left(T_{3,2}\right)$ & $(3,3,3,3,2,2,1,3,3,0)$ \\
\hline$C_{612}^{(1)}\left(T_{3,1}\right)$ & $(3,3,3,3,3,2,-1,0,0,2)$ \\
\hline$C_{612}^{(2)}\left(T_{3,2}\right)$ & $(3,3,3,3,1,1,0,1,3,3)$ \\
\hline$C_{613}\left(T_{3,2}\right)$ & $(3,3,3,3,2,1,0,3,3,2)$ \\
\hline$C_{621}\left(T_{3,1}\right)$ & $(3,3,3,3,1,0,0,3,0,3)$ \\
\hline$C_{628}\left(T_{3,2}\right)$ & $(3,3,3,3,3,2,-1,0,-1,2)$ \\
\hline
\end{tabular}


Table 4 (continued)

\begin{tabular}{|l|l|}
\hline extended quaternary forms & reduced quaternary forms \\
\hline$C_{637}\left(T_{3,2}\right)$ & $(3,3,3,3,1,1,-1,2,-3,0)$ \\
\hline$C_{640}\left(T_{3,2}\right)$ & $(3,3,3,3,1,1,1,3,3,2)$ \\
\hline$C_{648}^{(1)}\left(T_{3,1}\right)$ & $(3,3,3,3,0,0,0,3,3,0)$ \\
\hline$C_{660}\left(T_{3,2}\right)$ & $(3,3,3,3,3,2,-1,-1,-1,1)$ \\
\hline$C_{672}\left(T_{3,2}\right)$ & $(3,3,3,3,1,1,-1,3,0,3)$ \\
\hline$C_{685}\left(T_{3,2}\right)$ & $(3,3,3,3,3,2,-1,0,1,-1)$ \\
\hline$C_{688}\left(T_{3,2}\right)$ & $(3,3,3,3,3,2,-1,0,1,0)$ \\
\hline$C_{693}\left(T_{3,2}\right)$ & $(3,3,3,3,1,1,0,3,3,0)$ \\
\hline$C_{720}\left(T_{3,2}\right)$ & $(3,3,3,3,3,2,-1,0,0,0)$ \\
\hline
\end{tabular}

\subsection{Trials to Compute the Fourier Coefficients of Siegel Theta Series of Degree 4 for $\mathscr{L}$}

\subsubsection{First Case}

We specialize the general notations in Section 4.1 to the case $s=3$. For a fixed triple $\mathbf{x}_{1}, \mathbf{x}_{2}, \mathbf{x}_{3} \in \Lambda_{6}$ satisfying $\left[\mathbf{x}_{1}, \mathbf{x}_{2}, \mathbf{x}_{3}\right]=2 T_{3,1}$ we set

$$
\lambda_{a, b, c}\left(2 T_{3,1} ; \mathbf{x}_{1}, \mathbf{x}_{2}, \mathbf{x}_{3}\right)=\left|\left\{\mathbf{y} \in \Lambda_{6} \mid\left(\mathbf{x}_{1}, \mathbf{y}\right)=a,\left(\mathbf{x}_{2}, \mathbf{y}\right)=b,\left(\mathbf{x}_{3}, \mathbf{y}\right)=c\right\}\right| .
$$

Put $\boldsymbol{\alpha}=u \mathbf{x}_{1}+v \mathbf{x}_{2}+t \mathbf{x}_{3}$, where $u, v, t$ are algebraically independent real variables.

$$
\begin{aligned}
\sum_{\mathbf{y} \in \Lambda_{6}} & \lambda_{a, b, c}\left(2 T_{3,1} ; \mathbf{x}_{1}, \mathbf{x}_{2}, \mathbf{x}_{3}\right)(a u+b v+c t)^{2 k} \\
= & \sum_{\langle a, b, c\rangle \in C_{621}\left(T_{3,1},\langle 1,1,1\rangle\right)} \lambda_{a, b, c}\left(2 T_{3,1} ; \mathbf{x}_{1}, \mathbf{x}_{2}, \mathbf{x}_{3}\right)(a u+b v+c t)^{2 k} \\
& +\sum_{\langle a, b, c\rangle \in C_{612}\left(T_{3,1},\langle 1,1,0\rangle\right)} \lambda_{a, b, c}\left(2 T_{3,1} ; \mathbf{x}_{1}, \mathbf{x}_{2}, \mathbf{x}_{3}\right)(a u+b v+c t)^{2 k} \\
& +\sum_{\langle a, b, c\rangle \in C_{576}\left(T_{3,1},\langle 2,1,1\rangle\right)} \lambda_{a, b, c}\left(2 T_{3,1} ; \mathbf{x}_{1}, \mathbf{x}_{2}, \mathbf{x}_{3}\right)(a u+b v+c t)^{2 k} \\
& +\sum_{\langle a, b, c\rangle \in C_{549}\left(T_{3,1},\langle 2,2,1\rangle\right)} \lambda_{a, b, c}\left(2 T_{3,1} ; \mathbf{x}_{1}, \mathbf{x}_{2}, \mathbf{x}_{3}\right)(a u+b v+c t)^{2 k} \\
& +\sum_{\langle a, b, c\rangle \in C_{540}\left(T_{3,1},\langle 2,2,2\rangle\right)} \lambda_{a, b, c}\left(2 T_{3,1} ; \mathbf{x}_{1}, \mathbf{x}_{2}, \mathbf{x}_{3}\right)(a u+b v+c t)^{2 k} \\
& +\sum_{\langle a, b, c\rangle \in C_{504}\left(T_{3,1},\langle 2,2,0\rangle\right)} \lambda_{a, b, c}\left(2 T_{3,1} ; \mathbf{x}_{1}, \mathbf{x}_{2}, \mathbf{x}_{3}\right)(a u+b v+c t)^{2 k}
\end{aligned}
$$




$$
\begin{aligned}
& +\sum_{\langle a, b, c\rangle \in C_{477}\left(T_{3,1},\langle 3,2,2\rangle\right)} \lambda_{a, b, c}\left(2 T_{3,1} ; \mathbf{x}_{1}, \mathbf{x}_{2}, \mathbf{x}_{3}\right)(a u+b v+c t)^{2 k} \\
& +\sum_{\langle a, b, c\rangle \in C_{468}\left(T_{3,1},\langle 3,2,1\rangle\right)} \lambda_{a, b, c}\left(2 T_{3,1} ; \mathbf{x}_{1}, \mathbf{x}_{2}, \mathbf{x}_{3}\right)(a u+b v+c t)^{2 k} \\
& +\sum_{\langle a, b, c\rangle \in C_{432}\left(T_{3,1},\langle 3,3,2\rangle\right)} \lambda_{a, b, c}\left(2 T_{3,1} ; \mathbf{x}_{1}, \mathbf{x}_{2}, \mathbf{x}_{3}\right)(a u+b v+c t)^{2 k} \\
& +\sum_{\langle a, b, c\rangle \in C_{405,1}\left(T_{3,1},\langle 3,3,3\rangle\right)} \lambda_{a, b, c}\left(2 T_{3,1} ; \mathbf{x}_{1}, \mathbf{x}_{2}, \mathbf{x}_{3}\right)(a u+b v+c t)^{2 k} \\
& +\sum_{\langle a, b, c\rangle \in C_{405,2}\left(T_{3,1},\langle 3,3,1\rangle\right)} \lambda_{a, b, c}\left(2 T_{3,1} ; \mathbf{x}_{1}, \mathbf{x}_{2}, \mathbf{x}_{3}\right)(a u+b v+c t)^{2 k} \\
& +\sum_{\langle a, b, c\rangle \in C_{324}\left(T_{3,1},\langle 3,3,0\rangle\right)} \lambda_{a, b, c}\left(2 T_{3,1} ; \mathbf{x}_{1}, \mathbf{x}_{2}, \mathbf{x}_{3}\right)(a u+b v+c t)^{2 k} \\
& +\sum_{\langle a, b, c\rangle \in C_{0}\left(T_{3,1},\langle 6,3,3\rangle\right)} \lambda_{a, b, c}\left(2 T_{3,1} ; \mathbf{x}_{1}, \mathbf{x}_{2}, \mathbf{x}_{3}\right)(a u+b v+c t)^{2 k} \\
& =\rho_{k}\left(6 u^{2}+6 v^{2}+6 t^{2}+6 u v+6 u t+6 v t\right)^{k}, \quad 1 \leq k \leq 5 .
\end{aligned}
$$

By taking a sum over $\mathbf{x}_{1}, \mathbf{x}_{2}, \mathbf{x}_{3} \in \Lambda_{6}$ with $\left[\mathbf{x}_{1}, \mathbf{x}_{2}, \mathbf{x}_{3}\right]=2 T_{3,1}$ in the last equation we have

$$
\sum_{\substack{\mathbf{x}_{1}, \mathbf{x}_{2}, \mathbf{x}_{3} \in \Lambda_{6} \\\left[\mathbf{x}_{1}, \mathbf{x}_{2}, \mathbf{x}_{3}\right]=2 T_{3,1}}} \sum_{\substack{\langle a, b, c\rangle \in \\ C_{621}\left(T_{3,1},\langle 1,1,1\rangle\right)}} \lambda_{a, b, c}\left(2 T_{3,1} ; \mathbf{x}_{1}, \mathbf{x}_{2}, \mathbf{x}_{3}\right)(a u+b v+c t)^{2 k}
$$

$$
\begin{aligned}
& +\sum_{\substack{\mathbf{x}_{1}, \mathbf{x}_{2}, \mathbf{x}_{3} \in \Lambda_{6} \\
\left[\mathbf{x}_{1}, \mathbf{x}_{2}, \mathbf{x}_{3}\right]=2 T_{3,1}}} \sum_{\substack{\langle a, b, c\rangle \in \\
C_{612}\left(T_{3,1},\langle 1,1,0\rangle\right)}} \lambda_{a, b, c}\left(2 T_{3,1} ; \mathbf{x}_{1}, \mathbf{x}_{2}, \mathbf{x}_{3}\right)(a u+b v+c t)^{2 k} \\
& +\sum_{\substack{\mathbf{x}_{1}, \mathbf{x}_{2}, \mathbf{x}_{3} \in \Lambda_{6} \\
\left[\mathbf{x}_{1}, \mathbf{x}_{2}, \mathbf{x}_{3}\right]=2 T_{3,1}}} \sum_{\substack{\langle a, b, c\rangle \in \\
C_{576}\left(T_{3,1},\langle 2,1,1\rangle\right)}} \lambda_{a, b, c}\left(2 T_{3,1} ; \mathbf{x}_{1}, \mathbf{x}_{2}, \mathbf{x}_{3}\right)(a u+b v+c t)^{2 k} \\
& +\sum_{\substack{\left.\mathbf{x}_{1}, \mathbf{x}_{2}, \mathbf{x}_{3} \in \Lambda_{6} \\
\mathbf{x}_{1}, \mathbf{x}_{2}, \mathbf{x}_{3}\right]=2 T_{3,1}}} \sum_{\substack{\langle a, b, c\rangle \in \\
C_{549}\left(T_{3,1},\langle 2,2,1\rangle\right)}} \lambda_{a, b, c}\left(2 T_{3,1} ; \mathbf{x}_{1}, \mathbf{x}_{2}, \mathbf{x}_{3}\right)(a u+b v+c t)^{2 k}
\end{aligned}
$$$$
+\sum_{\substack{\mathbf{x}_{1}, \mathbf{x}_{2}, \mathbf{x}_{3} \in \Lambda_{6} \\\left[\mathbf{x}_{1}, \mathbf{x}_{2}, \mathbf{x}_{3}\right]=2 T_{3,1}}} \sum_{\substack{\langle a, b, c\rangle \in \\ C_{540}\left(T_{3,1},\langle 2,2,2\rangle\right)}} \lambda_{a, b, c}\left(2 T_{3,1} ; \mathbf{x}_{1}, \mathbf{x}_{2}, \mathbf{x}_{3}\right)(a u+b v+c t)^{2 k}
$$$$
+\sum_{\substack{\mathbf{x}_{1}, \mathbf{x}_{2}, \mathbf{x}_{3} \in \Lambda_{6} \\\left[\mathbf{x}_{1}, \mathbf{x}_{2}, \mathbf{x}_{3}\right]=2 T_{3,1}}} \sum_{\substack{\langle a, b, c\rangle \in \\ C_{504}\left(T_{3,1},\langle 2,2,0\rangle\right)}} \lambda_{a, b, c}\left(2 T_{3,1} ; \mathbf{x}_{1}, \mathbf{x}_{2}, \mathbf{x}_{3}\right)(a u+b v+c t)^{2 k}
$$$$
+\sum_{\substack{\mathbf{x}_{1}, \mathbf{x}_{2}, \mathbf{x}_{3} \in \Lambda_{6} \\\left[\mathbf{x}_{1}, \mathbf{x}_{2}, \mathbf{x}_{3}\right]=2 T_{3,1}}} \sum_{\substack{\langle a, b, c\rangle \in \\ C_{477}\left(T_{3,1},\langle 3,2,2\rangle\right)}} \lambda_{a, b, c}\left(2 T_{3,1} ; \mathbf{x}_{1}, \mathbf{x}_{2}, \mathbf{x}_{3}\right)(a u+b v+c t)^{2 k}
$$ 


$$
\begin{aligned}
& +\sum_{\substack{\mathbf{x}_{1}, \mathbf{x}_{2}, \mathbf{x}_{3} \in \Lambda_{6} \\
\left[\mathbf{x}_{1}, \mathbf{x}_{2}, \mathbf{x}_{3}\right]=2 T_{3,1}}} \sum_{\substack{\langle a, b, c\rangle \in \\
C_{468}\left(T_{3,1},\langle 3,2,1\rangle\right)}} \lambda_{a, b, c}\left(2 T_{3,1} ; \mathbf{x}_{1}, \mathbf{x}_{2}, \mathbf{x}_{3}\right)(a u+b v+c t)^{2 k} \\
& +\sum_{\substack{\mathbf{x}_{1}, \mathbf{x}_{2}, \mathbf{x}_{3} \in \Lambda_{6} \\
\left[\mathbf{x}_{1}, \mathbf{x}_{2}, \mathbf{x}_{3}\right]=2 T_{3,1}}} \sum_{\substack{\langle a, b, c\rangle \in \\
C_{432}\left(T_{3,1},\langle 3,3,2\rangle\right)}} \lambda_{a, b, c}\left(2 T_{3,1} ; \mathbf{x}_{1}, \mathbf{x}_{2}, \mathbf{x}_{3}\right)(a u+b v+c t)^{2 k} \\
& +\sum_{\substack{\mathbf{x}_{1}, \mathbf{x}_{2}, \mathbf{x}_{3} \in \Lambda_{6} \\
\left[\mathbf{x}_{1}, \mathbf{x}_{2}, \mathbf{x}_{3}\right]=2 T_{3,1}}} \sum_{\substack{\langle a, b, c\rangle \in \\
C_{405,1}\left(T_{3,1},\langle 3,3,3\rangle\right)}} \lambda_{a, b, c}\left(2 T_{3,1} ; \mathbf{x}_{1}, \mathbf{x}_{2}, \mathbf{x}_{3}\right)(a u+b v+c t)^{2 k} \\
& +\sum_{\substack{\mathbf{x}_{1}, \mathbf{x}_{2}, \mathbf{x}_{3} \in \Lambda_{6} \\
\left[\mathbf{x}_{1}, \mathbf{x}_{2}, \mathbf{x}_{3}\right]=2 T_{3,1}}} \sum_{\substack{\langle a, b, c\rangle \in \\
C_{405,2}\left(T_{3,1},\langle 3,3,1\rangle\right)}} \lambda_{a, b, c}\left(2 T_{3,1} ; \mathbf{x}_{1}, \mathbf{x}_{2}, \mathbf{x}_{3}\right)(a u+b v+c t)^{2 k} \\
& +\sum_{\substack{\mathbf{x}_{1}, \mathbf{x}_{2}, \mathbf{x}_{3} \in \Lambda_{6} \\
\left[\mathbf{x}_{1}, \mathbf{x}_{2}, \mathbf{x}_{3}\right]=2 T_{3,1}}} \sum_{\substack{\langle a, b, c\rangle \in \\
C_{324}\left(T_{3,1},\langle 3,3,0\rangle\right)}} \lambda_{a, b, c}\left(2 T_{3,1} ; \mathbf{x}_{1}, \mathbf{x}_{2}, \mathbf{x}_{3}\right)(a u+b v+c t)^{2 k} \\
& +\sum_{\substack{\mathbf{x}_{1}, \mathbf{x}_{2}, \mathbf{x}_{3} \in \Lambda_{6} \\
\left[\mathbf{x}_{1}, \mathbf{x}_{2}, \mathbf{x}_{3}\right]=2 T_{3,1}}} \sum_{\substack{\langle a, b, c\rangle \in \\
C_{0}\left(T_{3,1},\langle 6,3,3\rangle\right)}} \lambda_{a, b, c}\left(2 T_{3,1} ; \mathbf{x}_{1}, \mathbf{x}_{2}, \mathbf{x}_{3}\right)(a u+b v+c t)^{2 k} \\
& =\sum_{\substack{\mathbf{x}_{1}, \mathbf{x}_{2}, \mathbf{x}_{3} \in \Lambda_{6} \\
\left[\mathbf{x}_{1}, \mathbf{x}_{2}, \mathbf{x}_{3}\right]=2 T_{3,1}}} \rho_{k}\left(6 u^{2}+6 v^{2}+6 t^{2}+6 u v+6 u t+6 v t\right)^{k} .
\end{aligned}
$$

We observe that

$$
\begin{gathered}
\sum_{\substack{\mathbf{x}_{1}, \mathbf{x}_{2}, \mathbf{x}_{3} \in \Lambda_{6} \\
\left[\mathbf{x}_{1}, \mathbf{x}_{2}, \mathbf{x}_{3}\right]=2 T_{3,1}}} \sum_{\substack{\langle a, b, c\rangle \in \\
C_{621}\left(T_{3,1},\langle 1,1,1\rangle\right)}} \lambda_{a, b, c}\left(2 T_{3,1} ; \mathbf{x}_{1}, \mathbf{x}_{2}, \mathbf{x}_{3}\right)(a u+b v+c t)^{2 k} \\
=\sum_{\substack{\langle a, b, c\rangle \in \\
C_{621}\left(T_{3,1},\langle 1,1,1\rangle\right)}} \sum_{\substack{\mathbf{x}_{1}, \mathbf{x}_{2}, \mathbf{x}_{3} \in \Lambda_{6} \\
\left[\mathbf{x}_{1}, \mathbf{x}_{2}, \mathbf{x}_{3}\right]=2 T_{3,1}}} \lambda_{a, b, c}\left(2 T_{3,1} ; \mathbf{x}_{1}, \mathbf{x}_{2}, \mathbf{x}_{3}\right)(a u+b v+c t)^{2 k} \\
=\sum_{\substack{\langle a, b, c\rangle \in \\
C_{621}\left(T_{3,1},\langle 1,1,1\rangle\right)}} a\left(\left(T_{3,1},\{a / 2, b / 2, c / 2\}, 3\right), \mathscr{L}_{48}\right)(a u+b v+c t)^{2 k} . \\
=a\left(\left(T_{3,1},\{1 / 2,1 / 2,1 / 2\}, 3\right), \mathscr{L}_{48}\right) \sum_{\substack{\langle a, b, c\rangle \in \\
C_{621}\left(T_{3,1},\langle 1,1,1\rangle\right)}}(a u+b v+c t)^{2 k} .
\end{gathered}
$$

The last transition is certified by Proposition 2.4, since each triple $\langle a, b, c\rangle \in$ $C_{621}\left(T_{3,1},\langle 1,1,1\rangle\right)$ induces the integrally equivalent matrix $\left(T_{3,1},\{a / 2, b / 2, c / 2\}\right.$, 3) to each other. 
Other terms in (5.1) are treated in the same way. We rewrite (5.1) as

$$
\begin{aligned}
& a\left(\left(T_{3,1},\{1 / 2,1 / 2,1 / 2\}, 3\right), \mathscr{L}_{48}\right) \sum_{\substack{\langle a, b, c\rangle \in \\
C_{621}\left(T_{3,1},\langle 1,1,1\rangle\right)}}(a u+b v+c t)^{2 k} \\
& +a\left(\left(T_{3,1},\{1 / 2,1 / 2,0\}, 3\right), \mathscr{L}_{48}\right) \sum_{\substack{\langle a, b, c\rangle \in \\
C_{612}\left(T_{3,1},\langle 1,1,0\rangle\right)}}(a u+b v+c t)^{2 k} \\
& +a\left(\left(T_{3,1},\{1,1 / 2,1 / 2\}, 3\right), \mathscr{L}_{48}\right) \sum_{\substack{\langle a, b, c\rangle \in \\
C_{576}\left(T_{3,1},\langle 2,1,1\rangle\right)}}(a u+b v+c t)^{2 k} \\
& +a\left(\left(T_{3,1},\{1,1,1 / 2\}, 3\right), \mathscr{L}_{48}\right) \sum_{\substack{\langle a, b, c\rangle \in \\
C_{549}\left(T_{3,1},\langle 2,2,1\rangle\right)}}(a u+b v+c t)^{2 k} \\
& +a\left(\left(T_{3,1},\{1,1,1\}, 3\right), \mathscr{L}_{48}\right) \sum_{\substack{\langle a, b, c\rangle \in \\
C_{540}\left(T_{3,1},\langle 2,2,2\rangle\right)}}(a u+b v+c t)^{2 k} \\
& +a\left(\left(T_{3,1},\{1,1,0\}, 3\right), \mathscr{L}_{48}\right) \sum_{\substack{\langle a, b, c\rangle \in \\
C_{504}\left(T_{3,1},\langle 2,2,0\rangle\right)}}(a u+b v+c t)^{2 k} \\
& +a\left(\left(T_{3,1},\{3 / 2,1,1\}, 3\right), \mathscr{L}_{48}\right) \sum_{\substack{\langle a, b, c\rangle \in \\
C_{477}\left(T_{3,1},\langle 3,2,2\rangle\right)}}(a u+b v+c t)^{2 k} \\
& +a\left(\left(T_{3,1},\{3 / 2,1,1 / 2\}, 3\right), \mathscr{L}_{48}\right) \sum_{\substack{\langle a, b, c\rangle \in \\
C_{468}\left(T_{3,1},\langle 3,2,1\rangle\right)}}(a u+b v+c t)^{2 k} \\
& +a\left(\left(T_{3,1},\{3 / 2,3 / 2,1\}, 3\right), \mathscr{L}_{48}\right) \sum_{\substack{\langle a, b, c\rangle \in \\
C_{432}\left(T_{3,1},\langle 3,3,2\rangle\right)}}(a u+b v+c t)^{2 k} \\
& +a\left(\left(T_{3,1},\{3 / 2,3 / 2,3 / 2\}, 3\right), \mathscr{L}_{48}\right) \sum_{\substack{\langle a, b, c\rangle \in \\
C_{405,1}\left(T_{3,1},\langle 3,3,3\rangle\right)}}(a u+b v+c t)^{2 k} \\
& +a\left(\left(T_{3,1},\{3 / 2,3 / 2,1 / 2\}, 3\right), \mathscr{L}_{48}\right) \quad \sum_{\langle a, b, c\rangle \in}(a u+b v+c t)^{2 k} \\
& C_{405,2}\left(T_{3,1},\langle 3,3,1\rangle\right) \\
& +a\left(\left(T_{3,1},\{3 / 2,3 / 2,0\}, 3\right), \mathscr{L}_{48}\right) \sum_{\substack{\langle a, b, c\rangle \in \\
C_{324}\left(T_{3,1},\langle 3,3,0\rangle\right)}}(a u+b v+c t)^{2 k}
\end{aligned}
$$




$$
\begin{aligned}
& +\sum_{\substack{\mathbf{x}_{1}, \mathbf{x}_{2}, \mathbf{x}_{3} \in \Lambda_{6} \\
\left[\mathbf{x}_{1}, \mathbf{x}_{2}, \mathbf{x}_{3}\right]=2 T_{3,1}}} \sum_{\substack{\langle a, b, c\rangle \in \\
C_{0}\left(T_{3,1},\langle 6,3,3\rangle\right)}}(a u+b v+c t)^{2 k} \\
= & \sum_{\substack{\mathbf{x}_{1}, \mathbf{x}_{2}, \mathbf{x}_{3} \in \Lambda_{6} \\
\left[\mathbf{x}_{1}, \mathbf{x}_{2}, \mathbf{x}_{3}\right]=2 T_{3,1}}} \rho_{k}\left(6 u^{2}+6 v^{2}+6 t^{2}+6 u v+6 u t+6 v t\right)^{k} .
\end{aligned}
$$

The above equations are polynomial identities concerning the independent real variables $u, v, t$, and by comparing the coefficients we get many linear equations on the quantities $a\left(\left(T_{3,1},\{1 / 2,1 / 2,1 / 2\}, 3\right), \mathscr{L}_{48}\right), \ldots, a\left(\left(T_{3,1},\{3 / 2,3 / 2,0\}\right.\right.$, $\left.3), \mathscr{L}_{48}\right)$. The linear equations are not many enough to solve them separately, but we have strong relations among them. We summarize our effort as a proposition.

Proposition 5.3. Let $T_{4, *}$ be some indeces given in Table 5 below. Then the Fourier coefficients $a\left(T_{4, *}, L\right)$ are expressed as

(i) $\quad a\left(T_{4,1}, \mathscr{L}_{48}\right)=278 a\left(T_{3,1}, \mathscr{L}_{48}\right)-a\left(T_{4,3}, \mathscr{L}_{48}\right)$,

(ii) $a\left(T_{4,4}, \mathscr{L}_{48}\right)=-552 a\left(T_{3,1}, \mathscr{L}_{48}\right)+3 a\left(T_{4,3}, \mathscr{L}_{48}\right)$,

(iii) $a\left(T_{4,5}, \mathscr{L}_{48}\right)=1929 a\left(T_{3,1}, \mathscr{L}_{48}\right)-3 a\left(T_{4,3}, \mathscr{L}_{48}\right)$,

(iv) $a\left(T_{4,7}, \mathscr{L}_{48}\right)=8622 a\left(T_{3,1}, \mathscr{L}_{48}\right)-9 a\left(T_{4,3}, \mathscr{L}_{48}\right)$,

(v) $a\left(T_{4,8}, \mathscr{L}_{48}\right)=6768 a\left(T_{3,1}, \mathscr{L}_{48}\right)+9 a\left(T_{4,3}, \mathscr{L}_{48}\right)$,

(vi) $a\left(T_{4,11}, \mathscr{L}_{48}\right)=35064 a\left(T_{3,1}, \mathscr{L}_{48}\right)-18 a\left(T_{4,3}, \mathscr{L}_{48}\right)$,

(vii) $a\left(T_{4,17}, \mathscr{L}_{48}\right)=140952 a\left(T_{3,1}, \mathscr{L}_{48}\right)-30 a\left(T_{4,3}, \mathscr{L}_{48}\right)$,

(viii) $a\left(T_{4,18}, \mathscr{L}_{48}\right)=179352 a\left(T_{3,1}, \mathscr{L}_{48}\right)+36 a\left(T_{4,3}, \mathscr{L}_{48}\right)$,

(ix) $a\left(T_{4,21}, \mathscr{L}_{48}\right)=546237 a\left(T_{3,1}, \mathscr{L}_{48}\right)-54 a\left(T_{4,3}, \mathscr{L}_{48}\right)$,

(x) $a\left(T_{4,27}, \mathscr{L}_{48}\right)=1983762 a\left(T_{3,1}, \mathscr{L}_{48}\right)-99 a\left(T_{4,3}, \mathscr{L}_{48}\right)$,

(xi) $a\left(T_{4,29}, \mathscr{L}_{48}\right)=2647392 a\left(T_{3,1}, \mathscr{L}_{48}\right)+111 a\left(T_{4,3}, \mathscr{L}_{48}\right)$,

(xii) $a\left(T_{4,33}, \mathscr{L}_{48}\right)=6732912 a\left(T_{3,1}, \mathscr{L}_{48}\right)-164 a\left(T_{4,3}, \mathscr{L}_{48}\right)$.

\subsubsection{Second Case}

For a fixed triple $\mathbf{x}_{1}, \mathbf{x}_{2}, \mathbf{x}_{3} \in \Lambda_{6}$ satisfying $\left[\mathbf{x}_{1}, \mathbf{x}_{2}, \mathbf{x}_{3}\right]=2 T_{3,2}$ we set

$$
\lambda_{a, b, c}\left(2 T_{3,2} ; \mathbf{x}_{1}, \mathbf{x}_{2}, \mathbf{x}_{3}\right)=\left|\left\{\mathbf{y} \in \Lambda_{6} \mid\left(\mathbf{x}_{1}, \mathbf{y}\right)=a,\left(\mathbf{x}_{2}, \mathbf{y}\right)=b,\left(\mathbf{x}_{3}, \mathbf{y}\right)=c\right\}\right| .
$$


Put $\boldsymbol{\alpha}=u \mathbf{x}_{1}+v \mathbf{x}_{2}+t \mathbf{x}_{3}$, where $u, v, t$ are algebraically independent real variables. We verify that $(\boldsymbol{\alpha}, \boldsymbol{\alpha})=6 u^{2}+6 v^{2}+6 t^{2}+6 u v+6 u t+2 v t$ and $(\boldsymbol{a}, \mathbf{y})=a u+b v+c t$ with certain integers $a, b, c$. By applying the formulas $(2.1) \sim(2.5)$ we have

$$
\begin{aligned}
& \sum_{\mathbf{y} \in \Lambda_{6}} \lambda_{a, b, c}\left(2 T_{3,2} ; \mathbf{x}_{1}, \mathbf{x}_{2}, \mathbf{x}_{3}\right)(a u+b v+c t)^{2 k} \\
& =\sum_{\langle a, b, c\rangle \in C_{693}\left(T_{3,2},\langle 0,1,0\rangle\right)} \lambda_{a, b, c}\left(2 T_{3,2} ; \mathbf{x}_{1}, \mathbf{x}_{2}, \mathbf{x}_{3}\right)(a u+b v+c t)^{2 k} \\
& +\sum_{\langle a, b, c\rangle \in C_{688}\left(T_{3,2},\langle 1,1,0\rangle\right)} \lambda_{a, b, c}\left(2 T_{3,2} ; \mathbf{x}_{1}, \mathbf{x}_{2}, \mathbf{x}_{3}\right)(a u+b v+c t)^{2 k} \\
& +\sum_{\langle a, b, c\rangle \in C_{685}\left(T_{3,2},\langle 1,1,1\rangle\right)} \lambda_{a, b, c}\left(2 T_{3,2} ; \mathbf{x}_{1}, \mathbf{x}_{2}, \mathbf{x}_{3}\right)(a u+b v+c t)^{2 k} \\
& +\sum_{\langle a, b, c\rangle \in C_{672}\left(T_{3,2},\langle 0,1,-1\rangle\right)} \lambda_{a, b, c}\left(2 T_{3,2} ; \mathbf{x}_{1}, \mathbf{x}_{2}, \mathbf{x}_{3}\right)(a u+b v+c t)^{2 k} \\
& +\sum_{\langle a, b, c\rangle \in C_{660}\left(T_{3,2},\langle 0,1,1\rangle\right)} \lambda_{a, b, c}\left(2 T_{3,2} ; \mathbf{x}_{1}, \mathbf{x}_{2}, \mathbf{x}_{3}\right)(a u+b v+c t)^{2 k} \\
& +\sum_{\langle a, b, c\rangle \in C_{640}\left(T_{3,2},\langle 2,1,1\rangle\right)} \lambda_{a, b, c}\left(2 T_{3,2} ; \mathbf{x}_{1}, \mathbf{x}_{2}, \mathbf{x}_{3}\right)(a u+b v+c t)^{2 k} \\
& +\sum_{\langle a, b, c\rangle \in C_{637}\left(T_{3,2},\langle 1,2,0\rangle\right)} \lambda_{a, b, c}\left(2 T_{3,2} ; \mathbf{x}_{1}, \mathbf{x}_{2}, \mathbf{x}_{3}\right)(a u+b v+c t)^{2 k} \\
& +\sum_{\langle a, b, c\rangle \in C_{628}\left(T_{3,2},\langle 1,2,1\rangle\right)} \lambda_{a, b, c}\left(2 T_{3,2} ; \mathbf{x}_{1}, \mathbf{x}_{2}, \mathbf{x}_{3}\right)(a u+b v+c t)^{2 k} \\
& +\sum_{\langle a, b, c\rangle \in C_{613}\left(T_{3,2},\langle 2,2,1\rangle\right)} \lambda_{a, b, c}\left(2 T_{3,2} ; \mathbf{x}_{1}, \mathbf{x}_{2}, \mathbf{x}_{3}\right)(a u+b v+c t)^{2 k} \\
& +\sum_{\langle a, b, c\rangle \in C_{612}\left(T_{3,2},\langle 0,2,0\rangle\right)} \lambda_{a, b, c}\left(2 T_{3,2} ; \mathbf{x}_{1}, \mathbf{x}_{2}, \mathbf{x}_{3}\right)(a u+b v+c t)^{2 k} \\
& +\sum_{\langle a, b, c\rangle \in C_{597}\left(T_{3,2},\langle 0,2,-1\rangle\right)} \lambda_{a, b, c}\left(2 T_{3,2} ; \mathbf{x}_{1}, \mathbf{x}_{2}, \mathbf{x}_{3}\right)(a u+b v+c t)^{2 k} \\
& +\sum_{\langle a, b, c\rangle \in C_{592}\left(T_{3,2},\langle 2,2,0\rangle\right)} \lambda_{a, b, c}\left(2 T_{3,2} ; \mathbf{x}_{1}, \mathbf{x}_{2}, \mathbf{x}_{3}\right)(a u+b v+c t)^{2 k} \\
& +\sum_{\langle a, b, c\rangle \in C_{580}\left(T_{3,2},\langle 1,2,0\rangle\right)} \lambda_{a, b, c}\left(2 T_{3,2} ; \mathbf{x}_{1}, \mathbf{x}_{2}, \mathbf{x}_{3}\right)(a u+b v+c t)^{2 k} \\
& +\sum_{\langle a, b, c\rangle \in C_{573}\left(T_{3,2},\langle 0,2,1\rangle\right)} \lambda_{a, b, c}\left(2 T_{3,2} ; \mathbf{x}_{1}, \mathbf{x}_{2}, \mathbf{x}_{3}\right)(a u+b v+c t)^{2 k} \\
& +\sum_{\langle a, b, c\rangle \in C_{565}\left(T_{3,2},\langle 1,2,2\rangle\right)} \lambda_{a, b, c}\left(2 T_{3,2} ; \mathbf{x}_{1}, \mathbf{x}_{2}, \mathbf{x}_{3}\right)(a u+b v+c t)^{2 k} \\
& +\sum_{\langle a, b, c\rangle \in C_{532}\left(T_{3,2},\langle 2,3,1\rangle\right)} \lambda_{a, b, c}\left(2 T_{3,2} ; \mathbf{x}_{1}, \mathbf{x}_{2}, \mathbf{x}_{3}\right)(a u+b v+c t)^{2 k} \\
& +\sum_{\langle a, b, c\rangle \in C_{528}\left(T_{3,2},\langle 3,2,1\rangle\right)} \lambda_{a, b, c}\left(2 T_{3,2} ; \mathbf{x}_{1}, \mathbf{x}_{2}, \mathbf{x}_{3}\right)(a u+b v+c t)^{2 k} \\
& +\sum_{\langle a, b, c\rangle \in C_{525}\left(T_{3,2},\langle 3,2,2\rangle\right)} \lambda_{a, b, c}\left(2 T_{3,2} ; \mathbf{x}_{1}, \mathbf{x}_{2}, \mathbf{x}_{3}\right)(a u+b v+c t)^{2 k}
\end{aligned}
$$




$$
\begin{aligned}
& +\sum_{\langle a, b, c\rangle \in C_{517}\left(T_{3,2},\langle 2,3,0\rangle\right)} \lambda_{a, b, c}\left(2 T_{3,2} ; \mathbf{x}_{1}, \mathbf{x}_{2}, \mathbf{x}_{3}\right)(a u+b v+c t)^{2 k} \\
& +\sum_{\langle a, b, c\rangle \in C_{493}\left(T_{3,2},\langle 2,3,2\rangle\right)} \lambda_{a, b, c}\left(2 T_{3,2} ; \mathbf{x}_{1}, \mathbf{x}_{2}, \mathbf{x}_{3}\right)(a u+b v+c t)^{2 k} \\
& +\sum_{\langle a, b, c\rangle \in C_{480}\left(T_{3,2},\langle 0,2,2\rangle\right)} \lambda_{a, b, c}\left(2 T_{3,2} ; \mathbf{x}_{1}, \mathbf{x}_{2}, \mathbf{x}_{3}\right)(a u+b v+c t)^{2 k} \\
& +\sum_{\langle a, b, c\rangle \in C_{477}\left(T_{3,2},\langle 3,3,1\rangle\right)} \lambda_{a, b, c}\left(2 T_{3,2} ; \mathbf{x}_{1}, \mathbf{x}_{2}, \mathbf{x}_{3}\right)(a u+b v+c t)^{2 k} \\
& +\sum_{\langle a, b, c\rangle \in C_{468}\left(T_{3,2},\langle 3,3,2\rangle\right)} \lambda_{a, b, c}\left(2 T_{3,2} ; \mathbf{x}_{1}, \mathbf{x}_{2}, \mathbf{x}_{3}\right)(a u+b v+c t)^{2 k} \\
& +\sum_{\langle a, b, c\rangle \in C_{448}\left(T_{3,2},\langle 2,3,-1\rangle\right)} \lambda_{a, b, c}\left(2 T_{3,2} ; \mathbf{x}_{1}, \mathbf{x}_{2}, \mathbf{x}_{3}\right)(a u+b v+c t)^{2 k} \\
& +\sum_{\langle a, b, c\rangle \in C_{432}\left(T_{3,2},\langle 3,3,0\rangle\right)} \lambda_{a, b, c}\left(2 T_{3,2} ; \mathbf{x}_{1}, \mathbf{x}_{2}, \mathbf{x}_{3}\right)(a u+b v+c t)^{2 k} \\
& +\sum_{\langle a, b, c\rangle \in C_{405}\left(T_{3,2},\langle 3,3,3\rangle\right)} \lambda_{a, b, c}\left(2 T_{3,2} ; \mathbf{x}_{1}, \mathbf{x}_{2}, \mathbf{x}_{3}\right)(a u+b v+c t)^{2 k} \\
& +\sum_{\langle a, b, c\rangle \in C_{400}\left(T_{3,2},\langle 2,3,3\rangle\right)} \lambda_{a, b, c}\left(2 T_{3,2} ; \mathbf{x}_{1}, \mathbf{x}_{2}, \mathbf{x}_{3}\right)(a u+b v+c t)^{2 k} \\
& +\sum_{\langle a, b, c\rangle \in C_{0}\left(T_{3,2},\langle 6,3,3\rangle\right)} \lambda_{a, b, c}\left(2 T_{3,2} ; \mathbf{x}_{1}, \mathbf{x}_{2}, \mathbf{x}_{3}\right)(a u+b v+c t)^{2 k} \\
& =\rho_{k}\left(6 u^{2}+6 v^{2}+6 t^{2}+6 u v+6 u t+2 v t\right)^{k}, \quad 1 \leq k \leq 5 .
\end{aligned}
$$

From here the arguement is quite similar to that of Section 5.2.1 and we have

$$
\begin{aligned}
& a\left(\left(T_{3,2},\{0,1 / 2,0\}, 3\right), \mathscr{L}_{48}\right) \sum_{\substack{\langle a, b, c\rangle \in \\
C_{693}\left(T_{3,2},\langle 0,1,0\rangle\right)}}(a u+b v+c t)^{2 k} \\
& +a\left(\left(T_{3,2},\{1 / 2,1 / 2,0\}, 3\right), \mathscr{L}_{48}\right) \sum_{\substack{\langle a, b, c\rangle \in \\
C_{688}\left(T_{3,2},\langle 1,1,0\rangle\right)}}(a u+b v+c t)^{2 k} \\
& +a\left(\left(T_{3,2},\{1 / 2,1 / 2,1 / 2\}, 3\right), \mathscr{L}_{48}\right) \sum_{\substack{\langle a, b, c\rangle \in \\
C_{685}\left(T_{3,2},\langle 1,1,1\rangle\right)}}(a u+b v+c t)^{2 k} \\
& +a\left(\left(T_{3,2},\{0,1 / 2,-1 / 2\}, 3\right), \mathscr{L}_{48}\right) \sum_{\substack{\langle a, b, c\rangle \in \\
C_{672}\left(T_{3,2},\langle 0,1,-1\rangle\right)}}(a u+b v+c t)^{2 k} \\
& +a\left(\left(T_{3,2},\{0,1 / 2,1 / 2\}, 3\right), \mathscr{L}_{48}\right) \sum_{\substack{\langle a, b, c\rangle \in \\
C_{660}\left(T_{3,2},\langle 0,1,1\rangle\right)}}(a u+b v+c t)^{2 k}
\end{aligned}
$$


Siegel theta series for the 48-dimensional extremal lattices

$$
\begin{aligned}
& +a\left(\left(T_{3,2},\{1,1 / 2,1 / 2\}, 3\right), \mathscr{L}_{48}\right) \sum_{\substack{\langle a, b, c\rangle \in \\
C_{640}\left(T_{3,2},\langle 2,1,1\rangle\right)}}(a u+b v+c t)^{2 k} \\
& +a\left(\left(T_{3,2},\{1 / 2,1,0\}, 3\right), \mathscr{L}_{48}\right) \sum_{\substack{\langle a, b, c\rangle \in \\
C_{637}\left(T_{3,2},\langle 1,2,0\rangle\right)}}(a u+b v+c t)^{2 k} \\
& +a\left(\left(T_{3,2},\{1 / 2,1,1 / 2\}, 3\right), \mathscr{L}_{48}\right) \sum_{\substack{\langle a, b, c\rangle \in \\
C_{628}\left(T_{3,2},\langle 1,2,1\rangle\right)}}(a u+b v+c t)^{2 k} \\
& +a\left(\left(T_{3,2},\{1,1,1 / 2\}, 3\right), \mathscr{L}_{48}\right) \sum_{\substack{\langle a, b, c\rangle \in \\
C_{613}\left(T_{3,2},\langle 2,2,1\rangle\right)}}(a u+b v+c t)^{2 k} \\
& +a\left(\left(T_{3,2},\{0,1,0\}, 3\right), \mathscr{L}_{48}\right) \sum_{\substack{\langle a, b, c\rangle \in \\
C_{612}\left(T_{3,2},\langle 0,2,0\rangle\right)}}(a u+b v+c t)^{2 k} \\
& +a\left(\left(T_{3,2},\{0,1,-1 / 2\}, 3\right), \mathscr{L}_{48}\right) \sum_{\substack{\langle a, b, c\rangle \in \\
C_{597}\left(T_{3,2},\langle 0,2,-1\rangle\right)}}(a u+b v+c t)^{2 k} \\
& +a\left(\left(T_{3,2},\{1,1,0\}, 3\right), \mathscr{L}_{48}\right) \sum_{\substack{\langle a, b, c\rangle \in \\
C_{592}\left(T_{3,2},\langle 2,2,0\rangle\right)}}(a u+b v+c t)^{2 k} \\
& +a\left(\left(T_{3,2},\{1,1,1\}, 3\right), \mathscr{L}_{48}\right) \sum_{\substack{\langle a, b, c\rangle \in \\
C_{580}\left(T_{3,2},\langle 2,2,2\rangle\right)}}(a u+b v+c t)^{2 k} \\
& +a\left(\left(T_{3,2},\{1 / 2,1,0\}, 3\right), \mathscr{L}_{48}\right) \sum_{\substack{\langle a, b, c\rangle \in \\
C_{573}\left(T_{3,2},\langle 0,2,1\rangle\right)}}(a u+b v+c t)^{2 k} \\
& +a\left(\left(T_{3,2},\{1 / 2,1,1\}, 3\right), \mathscr{L}_{48}\right) \sum_{\substack{\langle a, b, c\rangle \in \\
C_{565}\left(T_{3,2},\langle 1,2,2\rangle\right)}}(a u+b v+c t)^{2 k} \\
& +a\left(\left(T_{3,2},\{1,3 / 2,1\}, 3\right), \mathscr{L}_{48}\right) \sum_{\substack{\langle a, b, c\rangle \in \\
C_{532}\left(T_{3,2},\langle 2,3,1\rangle\right)}}(a u+b v+c t)^{2 k} \\
& +a\left(\left(T_{3,2},\{3 / 2,1,1 / 2\}, 3\right), \mathscr{L}_{48}\right) \sum_{\substack{\langle a, b, c\rangle \in \\
C_{528}\left(T_{3,2},\langle 3,2,1\rangle\right)}}(a u+b v+c t)^{2 k} \\
& +a\left(\left(T_{3,2},\{3 / 2,1,1\}, 3\right), \mathscr{L}_{48}\right) \sum_{\substack{\langle a, b, c\rangle \in \\
C_{525}\left(T_{3,2},\langle 3,2,2\rangle\right)}}(a u+b v+c t)^{2 k}
\end{aligned}
$$




$$
\begin{aligned}
& +a\left(\left(T_{3,2},\{1,3 / 2,0\}, 3\right), \mathscr{L}_{48}\right) \sum_{\substack{\langle a, b, c\rangle \in \\
C_{517}\left(T_{3,2},\langle 2,3,0\rangle\right)}}(a u+b v+c t)^{2 k} \\
& +a\left(\left(T_{3,2},\{1,3 / 2,1\}, 3\right), \mathscr{L}_{48}\right) \sum_{\substack{\langle a, b, c\rangle \in \\
C_{493}\left(T_{3,2},\langle 2,3,2\rangle\right)}}(a u+b v+c t)^{2 k} \\
& +a\left(\left(T_{3,2},\{0,1,1\}, 3\right), \mathscr{L}_{48}\right) \sum_{\substack{\langle a, b, c\rangle \in \\
C_{480}\left(T_{3,2},\langle 0,2,2\rangle\right)}}(a u+b v+c t)^{2 k} \\
& +a\left(\left(T_{3,2},\{3 / 2,3 / 2,1 / 2\}, 3\right), \mathscr{L}_{48}\right) \sum_{\substack{\langle a, b, c\rangle \in \\
C_{477}\left(T_{3,2},\langle 3,3,1\rangle\right)}}(a u+b v+c t)^{2 k} \\
& +a\left(\left(T_{3,2},\{3 / 2,3 / 2,1\}, 3\right), \mathscr{L}_{48}\right) \sum_{\substack{\langle a, b, c\rangle \in \\
C_{468}\left(T_{3,2},\langle 3,3,2\rangle\right)}}(a u+b v+c t)^{2 k} \\
& +a\left(\left(T_{3,2},\{1,3 / 2,-1 / 2\}, 3\right), \mathscr{L}_{48}\right) \sum_{\substack{\langle a, b, c\rangle \in \\
C_{448}\left(T_{3,2},\langle 2,3,-1\rangle\right)}}(a u+b v+c t)^{2 k} \\
& +a\left(\left(T_{3,2},\{0,1,-1 / 2\}, 3\right), \mathscr{L}_{48}\right) \sum_{\substack{\langle a, b, c\rangle \in \\
C_{432}\left(T_{3,2},\langle 3,3,0\rangle\right)}}(a u+b v+c t)^{2 k} \\
& +a\left(\left(T_{3,2},\{3 / 2,3 / 2,3 / 2\}, 3\right), \mathscr{L}_{48}\right) \sum_{\substack{\langle a, b, c\rangle \in \\
C_{405}\left(T_{3,2},\langle 3,3,3\rangle\right)}}(a u+b v+c t)^{2 k} \\
& +a\left(\left(T_{3,2},\{1,3 / 2,3 / 2\}, 3\right), \mathscr{L}_{48}\right) \sum_{\substack{\langle a, b, c\rangle \in \\
C_{400}\left(T_{3,2},\langle 2,3,3\rangle\right)}}(a u+b v+c t)^{2 k} \\
& +a\left(T_{3,2}, \mathscr{L}_{48}\right) \sum_{\substack{\langle a, b, c\rangle \in \\
C_{0}\left(T_{3,2},\langle 6,3,3\rangle\right)}}(a u+b v+c t)^{2 k} \\
& =a\left(T_{3,2}, \mathscr{L}_{48}\right) \rho_{k}\left(6 u^{2}+6 v^{2}+6 t^{2}+6 u v+6 u t+2 v t\right)^{k}, \quad 1 \leq k \leq 5 .
\end{aligned}
$$

Proposition 5.4. Let $T_{4, *}$ be some indeces given in Table 5 below. Then the Fourier coefficients $a\left(T_{4, *}, L\right)$ are expressed as

$$
a\left(T_{4,2}, \mathscr{L}_{48}\right)=48 a\left(T_{3,2}, \mathscr{L}_{48}\right)-a\left(T_{4,4}, \mathscr{L}_{48}\right),
$$

(ii) $a\left(T_{4,5}, \mathscr{L}_{48}\right)=136 a\left(T_{3,2}, \mathscr{L}_{48}\right)-a\left(T_{4,4}, \mathscr{L}_{48}\right)$,

(iii) $a\left(T_{4,6}, \mathscr{L}_{48}\right)=183 a\left(T_{3,2}, \mathscr{L}_{48}\right)+2 a\left(T_{4,4}, \mathscr{L}_{48}\right)$, 
Siegel theta series for the 48-dimensional extremal lattices

(iv) $\quad a\left(T_{4,7}, \mathscr{L}_{48}\right)=688 a\left(T_{3,2}, \mathscr{L}_{48}\right)-3 a\left(T_{4,4}, \mathscr{L}_{48}\right)$,

(v) $\quad a\left(T_{4,8}, \mathscr{L}_{48}\right)=832 a\left(T_{3,2}, \mathscr{L}_{48}\right)+3 a\left(T_{4,4}, \mathscr{L}_{48}\right)$,

(vi) $\quad a\left(T_{4,9}, \mathscr{L}_{48}\right)=1152 a\left(T_{3,2}, \mathscr{L}_{48}\right)-4 a\left(T_{4,4}, \mathscr{L}_{48}\right)$,

(vii) $a\left(T_{4,10}, \mathscr{L}_{48}\right)=1824 a\left(T_{3,2}, \mathscr{L}_{48}\right)+a\left(T_{4,4}, \mathscr{L}_{48}\right)$,

(viii) $\quad a\left(T_{4,12}, \mathscr{L}_{48}\right)=5280 a\left(T_{3,2}, \mathscr{L}_{48}\right)-5 a\left(T_{4,4}, \mathscr{L}_{48}\right)$,

(ix) $\quad a\left(T_{4,13}, \mathscr{L}_{48}\right)=6912 a\left(T_{3,2}, \mathscr{L}_{48}\right)+9 a\left(T_{4,4}, \mathscr{L}_{48}\right)$,

(x) $a\left(T_{4,15}, \mathscr{L}_{48}\right)=8112 a\left(T_{3,2}, \mathscr{L}_{48}\right)-2 a\left(T_{4,4}, \mathscr{L}_{48}\right)$,

(xi) $\quad a\left(T_{4,14}, \mathscr{L}_{48}\right)=8334 a\left(T_{3,2}, \mathscr{L}_{48}\right)-9 a\left(T_{4,4}, \mathscr{L}_{48}\right)$,

(xii) $\quad a\left(T_{4,16}, \mathscr{L}_{48}\right)=9432 a\left(T_{3,2}, \mathscr{L}_{48}\right)+3 a\left(T_{4,4}, \mathscr{L}_{48}\right)$,

(xiii) $a\left(T_{4,19}, \mathscr{L}_{48}\right)=34752 a\left(T_{3,2}, \mathscr{L}_{48}\right)-7 a\left(T_{4,4}, \mathscr{L}_{48}\right)$,

(xiv) $\quad a\left(T_{4,20}, \mathscr{L}_{48}\right)=46464 a\left(T_{3,2}, \mathscr{L}_{48}\right)+11 a\left(T_{4,4}, \mathscr{L}_{48}\right)$,

(xv) $a\left(T_{4,22}, \mathscr{L}_{48}\right)=60672 a\left(T_{3,2}, \mathscr{L}_{48}\right)+2 a\left(T_{4,4}, \mathscr{L}_{48}\right)$,

(xvi) $\quad a\left(T_{4,24}, \mathscr{L}_{48}\right)=94362 a\left(T_{3,2}, \mathscr{L}_{48}\right)-2 a\left(T_{4,4}, \mathscr{L}_{48}\right)$,

(xvii) $a\left(T_{4,23}, \mathscr{L}_{48}\right)=93936 a\left(T_{3,2}, \mathscr{L}_{48}\right)+14 a\left(T_{4,4}, \mathscr{L}_{48}\right)$,

(xviii) $a\left(T_{4,25}, \mathscr{L}_{48}\right)=112896 a\left(T_{3,2}, \mathscr{L}_{48}\right)+4 a\left(T_{4,4}, \mathscr{L}_{48}\right)$,

(xix) $\quad a\left(T_{4,26}, \mathscr{L}_{48}\right)=192960 a\left(T_{3,2}, \mathscr{L}_{48}\right)-10 a\left(T_{4,4}, \mathscr{L}_{48}\right)$,

$(\mathrm{xx}) \quad a\left(T_{4,28}, \mathscr{L}_{48}\right)=199872 a\left(T_{3,2}, \mathscr{L}_{48}\right)-12 a\left(T_{4,4}, \mathscr{L}_{48}\right)$,

(xxi) $\quad a\left(T_{4,30}, \mathscr{L}_{48}\right)=335352 a\left(T_{3,2}, \mathscr{L}_{48}\right)+8 a\left(T_{4,4}, \mathscr{L}_{48}\right)$,

(xxii) $a\left(T_{4,31}, \mathscr{L}_{48}\right)=455712 a\left(T_{3,2}, \mathscr{L}_{48}\right)-2 a\left(T_{4,4}, \mathscr{L}_{48}\right)$,

(xxiii) $a\left(T_{4,32}, \mathscr{L}_{48}\right)=503577 a\left(T_{3,2}, \mathscr{L}_{48}\right)+18 a\left(T_{4,4}, \mathscr{L}_{48}\right)$,

(xxiv) $a\left(T_{4,34}, \mathscr{L}_{48}\right)=977472 a\left(T_{3,2}, \mathscr{L}_{48}\right)-22 a\left(T_{4,4}, \mathscr{L}_{48}\right)$,

$(\mathrm{xxv}) \quad a\left(T_{4,35}, \mathscr{L}_{48}\right)=1439112 a\left(T_{3,2}, \mathscr{L}_{48}\right)-2 a\left(T_{4,4}, \mathscr{L}_{48}\right)$,

(xxvi) $a\left(T_{4,36}, \mathscr{L}_{48}\right)=2172672 a\left(T_{3,2}, \mathscr{L}_{48}\right)+3 a\left(T_{4,4}, \mathscr{L}_{48}\right)$,

(xxvii) $a\left(T_{4,37}, \mathscr{L}_{48}\right)=2386992 a\left(T_{3,2}, \mathscr{L}_{48}\right)-7 a\left(T_{4,4}, \mathscr{L}_{48}\right)$,

(xxviii) $a\left(T_{4,38}, \mathscr{L}_{48}\right)=2788672 a\left(T_{3,2}, \mathscr{L}_{48}\right)+13 a\left(T_{4,4}, \mathscr{L}_{48}\right)$,

$\left(\right.$ xxix) $a\left(T_{4,39}, \mathscr{L}_{48}\right)=6344672 a\left(T_{3,2}, \mathscr{L}_{48}\right)-12 a\left(T_{4,4}, \mathscr{L}_{48}\right)$. 
REMARK 6. There are four overlapping cases in Proposition 5.3 and Proposition 5.4. Those are $a\left(T_{4,4}, \mathscr{L}_{48}\right), a\left(T_{4,5}, \mathscr{L}_{48}\right), a\left(T_{4,7}, \mathscr{L}_{48}\right)$ and $a\left(T_{4,8}, \mathscr{L}_{48}\right)$. Therefore these overlapping cases should be consistent. First we have $a\left(T_{4,4}, \mathscr{L}_{48}\right)=-552 a\left(T_{3,1}, \mathscr{L}_{48}\right)+3 a\left(T_{4,3}, \mathscr{L}_{48}\right)$. Next we have $a\left(T_{4,5}, \mathscr{L}_{48}\right)=$ $1929 a\left(T_{3,1}, \mathscr{L}_{48}\right)-3 a\left(T_{4,3}, \mathscr{L}_{48}\right)=136 a\left(T_{3,2}, \mathscr{L}_{48}\right)-a\left(T_{4,4}, \mathscr{L}_{48}\right)$. From these we must have $1929 a\left(T_{3,1}, \mathscr{L}_{48}\right)=136 a\left(T_{3,2}, \mathscr{L}_{48}\right)+552 a\left(T_{3,1}, \mathscr{L}_{48}\right)$. This equality is verified to be true by using the values $a\left(T_{3,1}, \mathscr{L}_{48}\right), a\left(T_{3,2}, \mathscr{L}_{48}\right)$ given in Section 4.3.1. Other overlapping cases are also verified to be true. By using $a\left(T_{4,4}, \mathscr{L}_{48}\right)=-552 a\left(T_{3,1}, \mathscr{L}_{48}\right)+3 a\left(T_{4,3}, \mathscr{L}_{48}\right)$ all $a\left(T_{4, j}, \mathscr{L}_{48}\right) 1 \leq j \leq 39$ can be expressed as linear functions of $a\left(T_{4,4}, \mathscr{L}_{48}\right)$ solely. This reflects Table 5 below.

\section{Some Mathematical Statements}

Salvati Manni [15] Theorem 3 showed

Theorem 6.1 (Salvati Manni). Let us assume $N=32,48$ (the dimensions of the lattices); then about the theta series associated to extremal lattices we can say

(i) it is unique in degree 3 ,

(ii) in degree 4 their difference is, up to a multiplicative constant (possibly 0), equal to a power of Schottky's polynomial J.

We normalize that the Fourier coefficient of $J^{3}$ at the index $T_{4,3}$ is 1 .

THEOREM 6.2. Let $\Theta_{4}\left(Z, \mathscr{L}_{48}\right)=\sum_{T} a\left(T, \mathscr{L}_{48}\right) e^{2 \pi i \sigma(T Z)}$ be the Fourier expansion of Siegel theta series of degree 4 for any even unimodular extremal 48dimensional lattice $\mathscr{L}_{48}$. Then we have

(1) $\Theta_{4}\left(Z, \mathscr{L}_{48}\right)$ is uniquely determined by the value of the Fourier coefficient at the index $T_{4,3}$ which is equivalent to $\left(T_{3,1},\{3,3,3\}, 3\right)$.

(2) The series defined by

$$
P \Theta_{4}(Z)=\Theta_{4}\left(Z, \mathscr{L}_{48}\right)-a\left(T_{4,3}, \mathscr{L}_{48}\right) J^{3}
$$

is a Siegel modular form of degree 4 and weight 16, and independent of the choice of extremal lattice $\mathscr{L}_{48}$.

Proof. (1) follows from (2). The latter part of (2) can be obtained from Salvati Manni's Theorem. Indeed, that Theorem says the equality

$$
\Theta_{4}\left(Z, \mathscr{L}_{48}^{(1)}\right)-\Theta_{4}\left(Z, \mathscr{L}_{48}^{(2)}\right)=c J^{3}
$$


holds for certain constant $c$. We compare the both sides of the above equation at the index $T_{4,3}$ in their Fourier expansions. The left hand equals $\left(a\left(T_{4,3}, \mathscr{L}_{48}^{(1)}\right)-\right.$ $\left.a\left(T_{4,3}, \mathscr{L}_{48}^{(2)}\right)\right)$ whereas the right hand equals $c$. This implies that

$$
\Theta_{4}\left(Z, \mathscr{L}_{48}^{(1)}\right)-\Theta_{4}\left(Z, \mathscr{L}_{48}^{(2)}\right)=\left(a\left(T_{4,3}, \mathscr{L}_{48}^{(1)}\right)-a\left(T_{4,3}, \mathscr{L}_{48}^{(2)}\right)\right) J^{3},
$$

or

$$
\Theta_{4}\left(Z, \mathscr{L}_{48}^{(1)}\right)-a\left(T_{4,3}, \mathscr{L}_{48}^{(1)}\right) J^{3}=\Theta_{4}\left(Z, \mathscr{L}_{48}^{(2)}\right)-a\left(T_{4,3}, \mathscr{L}_{48}^{(2)}\right) J^{3} .
$$

for any two 48-dimensional even unimodular extremal lattices $\mathscr{L}_{48}^{(1)}, \mathscr{L}_{48}^{(2)}$.

We call $\mathrm{P \Theta}_{4}(Z)$ a pan theta series in 48 dimension. As to the range of $a\left(T_{4,3}, \mathscr{L}_{48}\right)$ we have simple bounds.

THEOREM 6.3. Let $\mathscr{L}_{48}$ be any even unimodular 48 dimensional extremal lattice, and $a\left(T_{4,3}, \mathscr{L}_{48}\right)$ be the Fourier coefficients at the index $T_{4,3}$. Then we have the inequalities

$$
184 a\left(T_{3,1}, \mathscr{L}_{48}\right) \leq a\left(T_{4,3}, \mathscr{L}_{48}\right) \leq 278 a\left(T_{3,1}, \mathscr{L}_{48}\right) .
$$

Proof. These inequalities are derived from the non-negativities of $a\left(T_{4,1}, \mathscr{L}_{48}\right)$ and $a\left(T_{4,4}, \mathscr{L}_{48}\right)$ in Proposition 5.3.

Table 5. Fourier coefficients of the cube of Schottky modular form $J$ and the 48 dimensional pan theta series

\begin{tabular}{|r|l|r|r|}
\hline$D$ & \multicolumn{1}{|c|}{ reduced form } & \multicolumn{1}{|c|}{$J^{3}$} & \multicolumn{1}{|c|}{48 dim pan Theta } \\
\hline$* 324$ & $T_{4,1}=(3,3,3,3,0,0,0,3,3,3)$ & -1 & 889166157594624000 \\
400 & $T_{4,2}=(3,3,3,3,2,2,2,-3,-3,1)$ & -3 & 3319980113608704000 \\
$* 405$ & $T_{4,3}=(3,3,3,3,3,0,0,3,0,3)$ & 1 & 0 \\
405 & $T_{4,4}=(3,3,3,3,1,0,0,3,3,3)$ & 3 & -1765538557526016000 \\
432 & $T_{4,5}=(3,3,3,3,3,1,0,1,3,3)$ & -3 & 6169789633093632000 \\
448 & $T_{4,6}=(3,3,3,3,2,2,-2,1,1,3)$ & 6 & 2395231317513216000 \\
468 & $T_{4,7}=(3,3,3,3,1,1,0,3,3,3)$ & -9 & 27576944643096576000 \\
477 & $T_{4,8}=(3,3,3,3,2,1,0,0,3,3)$ & 9 & 21647037966188544000 \\
480 & $T_{4,9}=(3,3,3,3,2,0,0,2,-2,-3)$ & -12 & 44368751576088576000 \\
493 & $T_{4,10}=(3,3,3,3,3,2,0,1,2,3)$ & 3 & 57303240573616128000 \\
504 & $T_{4,11}=(3,3,3,3,3,2,0,3,0,0)$ & -18 & 112150079675891712000 \\
512 & $(3,3,3,3,2,2,-2,-2,-2,2)$ & 24 & \\
517 & $T_{4,12}=(3,3,3,3,3,2,-1,0,2,1)$ & -15 & 179816263956725760000 \\
525 & $T_{4,13}=(3,3,3,3,3,2,-1,2,-1,1)$ & 27 & 207949737058172928000 \\
528 & $T_{4,14}=(3,3,3,3,3,2,-1,2,2,2)$ & -6 & 266231700093026304000 \\
528 & $T_{4,15}=(3,3,3,3,3,2,-1,-1,2,-1)$ & -27 & 285779762192590848000 \\
529 & $(3,3,3,3,3,2,0,2,2,3)$ & -42 & \\
\hline
\end{tabular}


Table 5 (continued)

\begin{tabular}{|c|c|c|c|}
\hline$D$ & reduced form & $J^{3}$ & 48 dim pan Theta \\
\hline 532 & $T_{4,16}=(3,3,3,3,3,1,-1,-1,2,1)$ & 9 & 300151150097670144000 \\
\hline 540 & $T_{4,17}=(3,3,3,3,2,0,0,0,3,3)$ & -30 & 450826432536969216000 \\
\hline 544 & $(3,3,3,3,2,2,-1,0,1,3)$ & 12 & \\
\hline 549 & $T_{4,18}=(3,3,3,3,3,2,0,3,0,1)$ & 36 & 573646506103996416000 \\
\hline 565 & $T_{4,19}=(3,3,3,3,2,2,-1,3,3,1)$ & -21 & 1137774456506548224000 \\
\hline 573 & $T_{4,20}=(3,3,3,3,2,2,-1,3,3,0)$ & 33 & 1485278502155255808000 \\
\hline 576 & $(3,3,3,3,3,1,-1,2,1,3)$ & -72 & \\
\hline 576 & $T_{4,21}=(3,3,3,3,3,1,-1,3,0,1)$ & -54 & 1747105951172714496000 \\
\hline 576 & $(3,3,3,3,3,1,-1,-1,1,2)$ & -36 & \\
\hline 580 & $T_{4,22}=(3,3,3,3,3,2,-1,0,-2,2)$ & 6 & 1961283049773465600000 \\
\hline 585 & $(3,3,3,3,3,2,0,0,0,3)$ & -6 & \\
\hline 588 & $(3,3,3,3,2,2,0,1,-2,-2)$ & -48 & \\
\hline 589 & $(3,3,3,3,3,2,0,1,1,3)$ & 60 & \\
\hline 592 & $T_{4,23}=(3,3,3,3,3,2,0,-1,2,0)$ & 42 & 3017324585448456192000 \\
\hline 592 & $T_{4,24}=(3,3,3,3,3,2,-1,1,2,1)$ & -6 & 3059368871179106304000 \\
\hline 597 & $T_{4,25}=(3,3,3,3,2,2,1,3,3,0)$ & 12 & 3648984385676378112000 \\
\hline 605 & $(3,3,3,3,2,2,-1,-1,2,1)$ & 33 & \\
\hline 608 & $(3,3,3,3,2,2,-2,-1,2,-1)$ & -12 & \\
\hline 609 & $(3,3,3,3,1,0,0,2,-2,3)$ & 6 & \\
\hline 612 & $T_{4,26}=(3,3,3,3,3,2,-1,0,0,2)$ & -30 & 6266510441027665920000 \\
\hline 612 & $T_{4,27}=(3,3,3,3,1,1,0,1,3,3)$ & -99 & 6344942572382109696000 \\
\hline 613 & $T_{4,28}=(3,3,3,3,2,1,0,3,3,2)$ & -36 & 6493881102218625024000 \\
\hline 621 & $(3,3,3,3,3,1,-1,2,1,-2)$ & -45 & \\
\hline 621 & $(3,3,3,3,3,2,0,1,0,3)$ & -126 & \\
\hline 621 & $T_{4,29}=(3,3,3,3,1,0,0,3,0,3)$ & 111 & 8467522921894772736000 \\
\hline 628 & $T_{4,30}=(3,3,3,3,3,2,-1,0,-1,2)$ & 24 & 10845981623111491584000 \\
\hline 628 & $(3,3,3,3,2,2,1,0,3,2)$ & 54 & \\
\hline 636 & $(3,3,3,3,2,2,-1,0,3,0)$ & 18 & \\
\hline 637 & $T_{4,31}=(3,3,3,3,1,1,-1,2,-3,0)$ & -6 & 14761399210564091904000 \\
\hline 640 & $(3,3,3,3,2,2,0,2,2,3)$ & 144 & \\
\hline 640 & $T_{4,32}=(3,3,3,3,1,1,1,3,3,2)$ & 54 & 16276158128619777024000 \\
\hline 640 & $(3,3,3,3,3,1,0,0,1,3)$ & 34 & \\
\hline 640 & $(3,3,3,3,2,2,2,0,2,-2)$ & -24 & \\
\hline *648 & $T_{4,33}=(3,3,3,3,0,0,0,3,3,0)$ & -164 & 21534811123966672896000 \\
\hline 649 & $(3,3,3,3,3,2,0,0,2,1)$ & -54 & \\
\hline 660 & $T_{4,34}=(3,3,3,3,3,2,-1,-1,-1,1)$ & -66 & 31693489696333430784000 \\
\hline 661 & $(3,3,3,3,3,1,-1,0,2,1)$ & 12 & \\
\hline 664 & $(3,3,3,3,2,1,0,0,2,3)$ & -78 & \\
\hline 665 & $(3,3,3,3,2,2,1,-1,2,2)$ & 130 & \\
\hline 672 & $T_{4,35}=(3,3,3,3,1,1,-1,3,0,3)$ & -6 & 46608020590808162304000 \\
\hline 672 & $(3,3,3,3,3,2,0,2,0,2)$ & 48 & \\
\hline 672 & $(3,3,3,3,2,2,2,1,-2,-2)$ & -64 & \\
\hline 672 & $(3,3,3,3,2,1,0,2,-2,-1)$ & 24 & \\
\hline 676 & $(3,3,3,3,3,1,0,1,1,3)$ & -375 & \\
\hline 684 & $(3,3,3,3,2,2,0,3,0,0)$ & 60 & \\
\hline 685 & $T_{4,36}=(3,3,3,3,3,2,-1,0,1,-1)$ & 9 & 70354945978854211584000 \\
\hline 685 & $(3,3,3,3,2,1,0,1,-1,-3)$ & 42 & \\
\hline 688 & $T_{4,37}=(3,3,3,3,3,2,-1,0,1,0)$ & -21 & 77313182912338673664000 \\
\hline
\end{tabular}


Table 5 (continued)

\begin{tabular}{|l|l|r|r|}
\hline$D$ & \multicolumn{1}{|c|}{ reduced form } & $J^{3}$ & 48 dim pan Theta \\
\hline 688 & $(3,3,3,3,3,1,-1,0,-2,2)$ & -228 & \\
693 & $(3,3,3,3,1,1,-1,3,2,2)$ & 18 & \\
693 & $(3,3,3,3,3,1,-1,-1,-2,2)$ & 207 & \\
693 & $(3,3,3,3,3,1,0,0,0,3)$ & 267 & \\
693 & $T_{4,38}=(3,3,3,3,1,1,0,3,3,0)$ & 39 & 90285957229673447424000 \\
697 & $(3,3,3,3,3,2,0,2,2,1)$ & -146 & \\
700 & $(3,3,3,3,2,0,0,0,3,2)$ & 86 & \\
704 & $(3,3,3,3,2,2,-2,1,1,1)$ & 24 & \\
705 & $(3,3,3,3,2,2,-1,1,-2,0)$ & -98 & \\
705 & $(3,3,3,3,2,2,1,3,0,0)$ & -96 & \\
709 & $(3,3,3,3,2,1,0,1,3,2)$ & -197 & \\
712 & $(3,3,3,3,3,1,0,0,2,-2)$ & -288 & \\
713 & $(3,3,3,3,2,2,-1,1,2,-2)$ & 54 & \\
720 & $(3,3,3,3,2,2,2,-1,2,-1)$ & -54 & \\
720 & $(3,3,3,3,2,0,0,0,2,3)$ & 228 & \\
720 & $T_{4,39}=(3,3,3,3,3,2,-1,0,0,0)$ & -36 & 205488307640070733824000 \\
720 & $(3,3,3,3,2,2,2,3,1,1)$ & 468 & \\
720 & $(3,3,3,3,2,2,0,0,2,2)$ & 16 & \\
720 & $(3,3,3,3,1,1,1,-3,-2,1)$ & 198 & \\
& &
\end{tabular}

\section{Acknowledgment}

The author thanks the referees of the present article for reading carefully the initial draft and suggesting him some practical improvements in order to make the article more readable.

\section{References}

[1] The Brandt-Intrau tables of primitive positive-definite ternary quadratic forms, originally in http://www2.research.att.com/ njas/lattices/Brandt_1.html now in http://www.math.rwthaachen.de/ Gabriele.Nebe/LATTICES/Brandt_1.html.

[ 2 ] J. H. Conway and N. J. A. Sloane, Sphere Packings, Lattices and Groups, Springer-Verlag 1988. Third Edition 1998.

[3] E. Freitag, Siegelsche Modulfunktionen, Springer-Verlag 1983.

[4] E. Hecke, Analytische Arithmetik der positiven quadratischen Formen, Kgl. Danske Vid. Selskab. Mat.-fys. Medd. 13 (1940).

[5] J.-I. Igusa, Schottky's invariant and quadratic forms, Christoffel Sym., Birkhauser Verlag, 1981.

[ 6 ] C. L. Mallows, A. M. Odlyzko and N. J. A. Sloane, Upper bounds for modular forms, lattices, and codes, J. Alg. 36 (1975), 68-76.

[7] H. Minkowski, Gesamelte Abhandlungen, Chelsea, New-York, 1967.

[8] A Catalogue of Lattices in http://www.math.rwth-aachen.de/ Gabriele.Nebe/LATTICES/.

[9] G. Nipp, Tables of Quaternary Quadratic Forms (Computer Generated Tables). Available at http://www.math.rwth-aachen.de/ Gabriele.Nebe/LATTICES/nipp.html.

[10] M. Oura and M. Ozeki, Distinguishing Siegel theta series of degree 4 for the 32-dimensional even unimodular extremal lattices, Abhand. Math. Sem. Hamb. 86 (2016), 19-53. 
[11] M. Oura and M. Ozeki, A numerical study of Siegel theta series of various degrees for the 32-dimensional even unimodular extremal lattices. Kyushu J. Math. Vol. 70, No. 2 (2016), 281-314.

[12] M. Ozeki, On a property of Siegel theta-series. Mathematische Annalen Vol. 228 (1977), 249258.

[13] M. Ozeki, On the configurations of even unimodular lattices of rank 48. Arch. Math. 46 (1986), 247-287.

[14] M. Ozeki, Siegel Theta Series of Various Degrees for the Leech Lattice, Kyushu J. Math. 68 (2014), 53-91.

[15] R. Salvati Manni, Slopes of cusp forms and theta series, J. Num. Th. 83 (2000), 282-296.

[16] R. Scharlau and R. Schulze-Pillot, Extremal Lattices, in Algorithmic algebra and number theory, Springer (Heidelberg) 1997.

[17] A. Schiemann, Ein Beispiel positiv definiter quadratische Formen der Dimension 4 mit gleichen Darstellungszhlen, Arch. Math. 54 (1990), 372-375.

[18] A. Schiemann, Ternary positive definite quadratic forms are determined by their theta series, Math. Ann. 308 (1997), 507-517.

[19] B. Schöneberg, Das Verhalten von mehrfachen Thetareihen bei Modulsubstitutionen. Math. Ann. 116 (1939), 511-523.

[20] B. Schöneberg, Elliptic Modular Functions, Springer (1974).

[21] C. L. Siegel, Einführung in die Theorie der Modulfunktionen $n$-ten Grades, Math. Ann. 116 (1939), 617-657.

[22] C. L. Siegel, Lectures on Quadratic Forms, Tata Institute of Fundamental Research, Bombay (1967).

[23] B. L. van der Waerden and H. Gross, editors, Studien zur Theorie der quadratischen Formen, Birkhäuser, Basel, 1968.

Emeritus Professor at

Department of Mathematical Sciences

Facuty of Science, Yamagata University

Japan

E-mail: ozeki.mitio@ruby.plala.or.jp 